Homology, Homotopy and Applications, vol. 19(2), 2017, pp.61-82

\title{
COHOMOLOGY OF LINKING SYSTEMS WITH TWISTED COEFFICIENTS BY A $p$-SOLVABLE ACTION
}

\author{
RÉMI MOLINIER
}

\author{
(communicated by Nathalie Wahl)
}

\begin{abstract}
In this paper, we study the cohomology of the geometric realization of linking systems with twisted coefficients. More precisely, given a prime $p$ and a $p$-local finite group $(S, \mathcal{F}, \mathcal{L})$, we compare the cohomology of $\mathcal{L}$ with twisted coefficients with the submodule of $\mathcal{F}^{c}$-stable elements in the cohomology of $S$. We start with the particular case of constrained fusion systems. Then, we study the case of $p$-solvable actions on the coefficients.
\end{abstract}

\section{Introduction}

The notion of saturated fusion system was introduced by Puig in the 1990s in a context of modular representation theory. Since their introduction, topologists use them to study classifying spaces of finite groups or, more precisely, their $p$-completions. A $p$-local finite group is a triple $(S, \mathcal{F}, \mathcal{L})$ where $S$ is a $p$-group, $\mathcal{F}$ a saturated fusion system over $S$ and $\mathcal{L}$ an associated linking system. For a $p$-local finite group $(S, \mathcal{F}, \mathcal{L})$, $|\mathcal{L}|_{p}^{\wedge}$ is called its classifying space. The theory of $p$-local finite groups have been studied in details by Broto, Levi, Oliver and others (see [BLO2, OV1, 5a1, 5a2]). The linking system and its geometric realization, even without $p$-completion, play here a fundamental and central role. In fact, for a given saturated fusion systems, the existence and uniqueness of an associated linking system were shown more recently by Chermak $[\mathbf{C h}]$ (using the theory of partial groups). The proof of this important conjecture highlights that linking systems and their geometric realizations form a deep link between fusion system theory and homotopy theory (we refer to Aschbacher, Kessar and Oliver [AKO] for more details about fusion systems in general).

An old and well-known result due to Cartan and Eilenberg (see [CE, Theorem XII.10.1]) expresses the cohomology of a finite group $G$ in a $\mathbb{Z}_{(p)}[G]$-module as the submodule of "stable" elements in the cohomology of a Sylow $p$-subgroup of $G$. This submodule of stable elements corresponds to the inverse limit over the "fusion" of the group cohomology functor. One important result in the theory of $p$ local finite groups is an analog of this theorem for $p$-local finite groups which tells us that the cohomology of the geometric realization of a linking system can be computed by $\mathcal{F}$-stable elements. More precisely, there is a natural inclusion of $B S$ into $|\mathcal{L}|$ and

Received February 15, 2016, revised November 3, 2016; published on August 9, 2017. 2010 Mathematics Subject Classification: 55R40, 55N25, 55R35, 20J06, 20D20, 20J15.

Key words and phrases: fusion system, $p$-local finite group, cohomology with twisted coefficients, group cohomology.

Article available at http://dx.doi.org/10.4310/HHA.2017.v19.n2.a4

Copyright (C) 2017, International Press. Permission to copy for private use granted. 
it induces the following isomorphism. Here, $\mathcal{F}^{c}$ is the full subcategory of $\mathcal{F}$ consisting of $\mathcal{F}$-centric subgroups of $S$ and, for $A$ a finite $\mathbb{Z}_{(p)}$-module, $H^{*}\left(\mathcal{F}^{c}, A\right) \subseteq H^{*}(S, A)$ is the submodule of $\mathcal{F}$-stable elements.

Theorem 1.1. Let $(S, \mathcal{F}, \mathcal{L})$ be a p-local finite group and $A$ be a finite $\mathbb{Z}_{(p)}$-module. The inclusion of $B S$ in $|\mathcal{L}|$ induces a natural isomorphism

$$
H^{*}\left(|\mathcal{L}|_{p}^{\wedge}, A\right) \cong H^{*}(|\mathcal{L}|, A) \stackrel{\cong}{\longrightarrow} H^{*}\left(\mathcal{F}^{c}, A\right) .
$$

Proof. The case $A=\mathbb{F}_{p}$ is $[$ BLO2, Theorem B] and the general case is proven in [5a2, Lemma 6.12].

One question asked by Oliver in his book with Aschbacher and Kessar [AKO] is the understanding of the cohomology of $|\mathcal{L}|$ with twisted coefficients. Indeed, this cohomology appears for example in the study of extensions of $p$-local finite groups or, more directly, can give more information about the link between the fusion system and the spaces $|\mathcal{L}|$ or $|\mathcal{L}|_{p}^{\wedge}$. Recall that, if a space $X$ has a universal covering space $\widetilde{X}$, the cohomology of $X$ with twisted coefficients in a $\mathbb{Z}\left[\pi_{1}(X)\right]$-module $M$ is the cohomology of the chain complex

$$
C^{*}(X ; M)=\operatorname{Hom}_{\mathbb{Z}\left[\pi_{1}(X)\right]}\left(S_{*}(\tilde{X}), M\right),
$$

where $S_{*}(\tilde{X})$ is the usual singular chain complex of $\widetilde{X}$.

Levi and Ragnarsson $[\mathbf{L R}]$ already give some tools along these lines. In an other paper [Mo1], the author extends Theorem 1.1 to the case of nilpotent actions on the coefficients. The main ingredient is to construct, as in the trivial coefficient case, an idempotent of $H^{*}(S, M)$ with image $H^{*}\left(\mathcal{F}^{c}, M\right)$.

In this paper, we also want to extend Theorem 1.1 to twisted coefficients but when the action factors through a $p$-solvable group. The methods used here are completely different from the ones used in [Mo1] and also more direct. We first have a look at constrained fusion systems. In that case we are able to prove that, with any coefficient module, the cohomology of $|\mathcal{L}|$ can be computed by stable elements.

Theorem A (see Corollary 3.5). Let $(S, \mathcal{F}, \mathcal{L})$ be a p-local finite group. If $\mathcal{F}$ is constrained and $M$ is a $\mathbb{Z}_{(p)}\left[\pi_{1}(|\mathcal{L}|)\right]$-module, then the inclusion of $B S$ in $|\mathcal{L}|$ induces an isomorphism,

$$
H^{*}(|\mathcal{L}|, M) \cong H^{*}\left(\mathcal{F}^{c}, M\right)
$$

Next we focus on $p$-solvable actions. The main ingredients here are $p$-local finite subgroups of index a power of $p$ or prime to $p$ and their homotopy properties. We start by looking at $p$-local subgroups of index prime to $p$ (see Definition 2.6(b)).

Theorem B (see Theorem 4.3). Let $(S, \mathcal{F}, \mathcal{L})$ be a p-local finite group and denote by $\left(S, O^{p^{\prime}}(\mathcal{F}), O^{p^{\prime}}(\mathcal{L})\right)$ its minimal p-local subgroup of index prime to $p$. If $M$ is a $\mathbb{Z}_{(p)}\left[\pi_{1}(|\mathcal{L}|)\right]$-module and if the inclusion of $B S$ in $\left|O^{p^{\prime}}(\mathcal{L})\right|$ induces an isomorphism

$$
H^{*}\left(\left|O^{p^{\prime}}(\mathcal{L})\right|, M\right) \cong H^{*}\left(O^{p^{\prime}}(\mathcal{F})^{c}, M\right),
$$

then the inclusion of $B S$ in $|\mathcal{L}|$ induces an isomorphism

$$
H^{*}(|\mathcal{L}|, M) \cong H^{*}\left(\mathcal{F}^{c}, M\right)
$$


This theorem allows us to prove that if the action on the coefficients factor through a $p^{\prime}$-group or, even better, a $p$-nilpotent group, then the cohomology of $|\mathcal{L}|$ can be computed by stable elements.

It is much more complicated to work with $p$-local finite groups of index a power of $p$, especially on the level of stable elements. Indeed, for $\left(S_{0}, \mathcal{F}_{0}, \mathcal{L}_{0}\right)$ a $p$-local subgroup of $(S, \mathcal{F}, \mathcal{L})$ of index a power of $p$ and $M$ a $\mathbb{Z}_{(p)}\left[\pi_{1}(|\mathcal{L}|)\right]$-module, it is difficult to compare $H^{*}\left(\mathcal{F}^{c}, M\right)$ and $H^{*}\left(\mathcal{F}_{0}^{c}, M\right)$. The difficulty mostly comes from the fact that we are working on different $p$-groups: $S$ and $S_{0}$. But when we work with a $p$-local finite group realizable by a finite group $G$, and if $G$ acts "consistently" on the coefficients it is possible to get some positive results (see Section 5).

Theorem C (see Corollary 5.5). Let $G$ be a finite group, $S$ a Sylow p-subgroup of $G$ and $(S, \mathcal{F}, \mathcal{L})$ the associated $p$-local finite group. Let $M$ be a $\mathbb{Z}_{(p)}\left[\pi_{1}(|\mathcal{L}|)\right]$-module and assume that $G$ acts consistently on $M$. If both actions factor through a given $p$-solvable $\Gamma$ and all the $M$-essential subgroups (see Definition 5.3) of $S$ are p-centric, then we have natural isomorphisms,

$$
H^{*}(|\mathcal{L}|, M) \cong H^{*}(G, M) \cong H^{*}\left(\mathcal{F}^{c}, M\right) .
$$

All of these results lead us to the following conjecture.

Conjecture A (see Conjecture 5.6). Let $(S, \mathcal{F}, \mathcal{L})$ be a p-local finite group and $M a$ $\mathbb{Z}_{(p)}\left[\pi_{1}(|\mathcal{L}|)\right]$-module. If the action of $\pi_{1}(|\mathcal{L}|)$ on $M$ is p-solvable, then the inclusion of $B S$ in $|\mathcal{L}|$ induces a natural isomorphism

$$
H^{*}(|\mathcal{L}|, M) \stackrel{\cong}{\longrightarrow} H^{*}\left(\mathcal{F}^{c}, M\right)
$$

We finish this paper with an example for Conjecture 5.6 which does not follow from the other results.

\section{Organization}

In Section 2, we give some background on $p$-local finite groups and stable elements. Section 3 is dedicated to the case of constrained fusion systems, Section 4 to coprime actions and Section 5 to $p$-solvable actions for a realizable $p$-local finite group. Finally, we give in Section 6 an example for Conjecture 5.6.

\section{Acknowledgments}

I would like to thank Bob Oliver, my $\mathrm{PhD}$ adviser, for his help and support all along this work. I am also grateful to Jesper Grodal for his hospitality at the Center for Symmetry and Deformation and many fruitful conversations. Finally, I would like to thank the referee for his very careful reading of the paper and all his useful and accurate comments.

\section{Background}

We give here a very short introduction to $p$-local finite groups. The notion of fusion system was first introduced by Puig for modular representation theory purpose. Later, 
Broto, Levi and Oliver developed the notion of linking systems and $p$-local finite groups to study $p$-completed classifying spaces of finite groups and spaces which have similar homotopy properties. We refer the reader interested in more details to Aschbacher, Kessar and Oliver [AKO].

\subsection{Fusion systems and linking systems}

A fusion system over a $p$-group $S$ is a way to abstract the action of a finite group $G$ with $S \in \operatorname{Syl}_{p}(G)$ on the subgroups of $S$ by conjugation. For $G$ a finite group and $g \in G$, we will denote by $c_{g}$ the homomorphism $x \in G \mapsto g x g^{-1} \in G$ and for $H, K$ two subgroups of $G, \operatorname{Hom}_{G}(H, K)$ will denote the set of all group homomorphism $c_{g}$ for $g \in G$ such that $c_{g}(H) \leqslant K$.

Definition 2.1. Let $S$ be a finite $p$-group. A fusion system over $S$ is a small category $\mathcal{F}$, where $\operatorname{Ob}(\mathcal{F})$ is the set of all subgroups of $S$ and which satisfies the following two properties for all $P, Q \leqslant S$ :

(a) $\operatorname{Hom}_{S}(P, Q) \subseteq \operatorname{Mor}_{\mathcal{F}}(P, Q) \subseteq \operatorname{Inj}(P, Q)$;

(b) each $\varphi \in \operatorname{Mor}_{\mathcal{F}}(P, Q)$ is the composite of an $\mathcal{F}$-isomorphism followed by an inclusion.

A fusion system is saturated if it satisfy two more technical axioms called the saturation axioms (we refer the reader to [AKO, Definition I.2.1] for a proper definition).

The composition in a fusion system is given by composition of homomorphisms. We usually write $\operatorname{Hom}_{\mathcal{F}}(P, Q)=\operatorname{Mor}_{\mathcal{F}}(P, Q)$ to emphasize that the morphisms in $\mathcal{F}$ are homomorphisms. For $P, Q \leqslant S$, we say that $P$ is $\mathcal{F}$-conjugate to $Q$ if there is an $\mathcal{F}$-isomorphism between $P$ and $Q$. We denote by $P^{\mathcal{F}}$ the set of all subgroups of $S$ which are $\mathcal{F}$ conjugate to $P$.

The typical example of a saturated fusion system is the fusion system $\mathcal{F}_{S}(G)$ of a finite group $G$ over $S \in \operatorname{Syl}_{p}(G)$.

For the purpose of this paper, we need to distinguish some collections of subgroups of $S$.

Definition 2.2. Let $\mathcal{F}$ be a saturated fusion system over a finite $p$-group $S$.

(a) A subgroup $P \leqslant S$ is $\mathcal{F}$-centric if for every $Q \in P^{\mathcal{F}}, C_{S}(Q)=Z(Q)$.

(b) A subgroup $P \leqslant S$ is $\mathcal{F}$-radical if $O_{p}\left(\operatorname{Aut}_{\mathcal{F}}(P)\right)=\operatorname{Inn}(P)$.

(c) A subgroup $P \leqslant S$ is $\mathcal{F}$-quasicentric if for each $Q \leqslant P C_{S}(P)$ containing $P$, and each $\alpha \in \operatorname{Aut}_{\mathcal{F}}(Q)$ such that $\left.\alpha\right|_{P}=\mathrm{Id}, \alpha$ has a $p$-power order.

We let $\mathcal{F}^{c r} \subseteq \mathcal{F}^{c} \subseteq \mathcal{F}^{q} \subseteq \mathcal{F}$ denote the full subcategories of $\mathcal{F}$ with objects the $\mathcal{F}$ centric and $\mathcal{F}$-radical subgroups, the $\mathcal{F}$-centric subgroups and the $\mathcal{F}$-quasicentric subgroups, respectively.

If $\mathcal{F}=\mathcal{F}_{S}(G)$, a subgroup $P \leqslant S$ is

(a) $\mathcal{F}$-centric if and only if it is $p$-centric (i.e. $Z(P) \in \operatorname{Syl}_{p}\left(C_{G}(P)\right)$ ),

(b) $\mathcal{F}$-radical if $P / Z(P)=O_{p}\left(N_{G}(P) / C_{G}(P)\right)$.

(c) $\mathcal{F}$-quasicentric if and only if $O^{p}\left(C_{G}(P)\right)$ has order prime to $p$. 
The notion of linking system has been introduced by Broto, Levi and Oliver [BLO2] and generalized by Broto, Castellana, Grodal and Oliver in [5a1]. We refer the reader to theses papers, or [AKO, Part III], for a proper definition. We recall here some basic facts about linking systems which will be needed here.

For $G$ a finite group, $S \in \operatorname{Syl}_{p}(G)$ and $\mathcal{H}$ a collection of subgroups of $S$, the transporter category of $G$ over $S$ with set of objects $\mathcal{H}$ is the category $\mathcal{T}_{H}^{\mathcal{H}}(G)$ with objects $\mathcal{H}$ and for $P, Q \in \mathcal{H}, \operatorname{Mor}_{\mathcal{L}}(P, Q)=T_{G}(P, Q)=\left\{g \in G \mid P^{g} \leqslant Q\right\}$. For $\mathcal{F}$ a saturated fusion system over a $p$-group $S$, a linking system associated to $\mathcal{F}$ is a certain finite category with objects a collection $\mathcal{H}$ of subgroups of $S$ together with two functors

$$
\mathcal{T}_{S}^{\mathcal{H}}(S) \stackrel{\delta}{\longrightarrow} \mathcal{L} \stackrel{\pi}{\longrightarrow} \mathcal{F} .
$$

$\delta$ is the identity on objects and injective on morphisms and $\pi$ is injective on objects and surjective on morphisms. The collection $\mathcal{H}$ has to be stable by overgroups and $\mathcal{F}$-conjugation and the following proposition tell you which collection you can have.

Proposition 2.3. Let $\mathcal{F}$ be a saturated fusion system over a p-group $S$. Let $\mathcal{L}$ be a linking system associated to $\mathcal{F}$.

(a) $O b\left(\mathcal{F}^{c r}\right) \subseteq O b(\mathcal{L}) \subseteq O b\left(\mathcal{F}^{q}\right)$, and there exists a linking system $\mathcal{L}^{q}$ associated to $\mathcal{F}$ such that $O b\left(\mathcal{L}^{q}\right)=O b\left(\mathcal{F}^{q}\right)$, and $\mathcal{L}$ is a full subcategory of $\mathcal{L}^{q}$.

(b) For every subset $O b\left(\mathcal{F}^{c r}\right) \subseteq \mathcal{H} \subseteq O b\left(\mathcal{F}^{q}\right)$ stable by $\mathcal{F}$-conjugacy and overgroups, the full subcategory $\mathcal{L}^{\mathcal{H}}$ of $\mathcal{L}^{q}$ with set of objects $\mathcal{H}$ is also a linking system associated to $\mathcal{F}$.

Proof. The first point of (a) can be found for example in [O4, Proposition 4(g)]. For the second statement of (a), you can find a proof in [AKO, Proposition III.4.8]. Finally, (b) is a consequence of the definition of linking systems.

If $\mathcal{H}=\mathrm{Ob}\left(\mathcal{F}^{q}\right), \mathcal{L}$ is called a quasicentric linking system and if $\mathcal{H}=\mathrm{Ob}\left(\mathcal{F}^{c}\right), \mathcal{L}$ is called a centric linking system.

Definition 2.4. A $p$-local finite group is a triple $(S, \mathcal{F}, \mathcal{L})$ where $\mathcal{F}$ is a saturated fusion system over $S$ and $\mathcal{L}$ is an associated linking system. If $\left(S_{0}, \mathcal{F}_{0}, \mathcal{L}_{0}\right)$ is an other $p$-local finite group, we will say that $\left(S_{0}, \mathcal{F}_{0}, \mathcal{L}_{0}\right)$ is a $p$-local subgroup of $(S, \mathcal{F}, \mathcal{L})$ if $S_{0} \leqslant S$ and $\mathcal{F}_{0} \subseteq \mathcal{F}$ is a subsystem of $\mathcal{F}$. Notice that we do not require that $\mathcal{L}_{0}$ is a subcategory of $\mathcal{L}$.

The typical example you should have in mind is the following. For $G$ a finite group and $S \in \operatorname{Syl}_{p}(G)$ let $\mathcal{L}_{S}^{q}(G)$ be the category with objects the $\mathcal{F}_{S}(G)$-quasicentric subgroups of $G$ and, for $P, Q \in \mathrm{Ob}(\mathcal{L})$,

$$
\operatorname{Mor}_{\mathcal{L}}(P, Q)=T_{G}(P, Q) / O^{p}\left(C_{G}(P)\right) .
$$

Then $\left(S, \mathcal{F}_{S}(G), \mathcal{L}_{S}^{q}(G)\right)$ defines a $p$-local finite group where $\mathcal{L}_{S}^{q}(G)$ is a quasicentric linking system. We also denote by $\mathcal{L}_{S}^{c}(G)$ the full subcategory of $\mathcal{L}_{S}^{q}(G)$ with objects the $p$-centric subgroups of $S$ and it is a centric linking system.

We finish with some basic homotopy properties about linking systems which will be needed in this paper. We refer the reader interested in more details to $[\mathbf{A K O}$, Part III]. For $(S, \mathcal{F}, \mathcal{L})$ a $p$-local finite group, we write $|\mathcal{L}|$ for the geometric realization of $\mathcal{L}$ and $\pi_{\mathcal{L}}=\pi_{1}(|\mathcal{L}|)$ for its fundamental group. The following theorem will allow us to change the set of objects of $\mathcal{L}$ without changing the homotopy type of $|\mathcal{L}|$. 
Theorem 2.5 ([5a1, Theorem 3.5]). Let $\mathcal{F}$ be a saturated fusion system over a $p$ group $S$. Let $\mathcal{L}_{0} \subseteq \mathcal{L}$ be two linking systems associated to $\mathcal{F}$ with a different set of objects. Then the inclusion induces a homotopy equivalence of space $\left|\mathcal{L}_{0}\right| \simeq|\mathcal{L}|$.

\section{2. $\quad p$-local finite subgroups of index a power of $p$ or prime to $p$}

The notions $p$-local subgroups of index a power of $p$ or prime to $p$ have been introduced and studied by Broto, Castellana, Grodal, Levi and Oliver [5a2]. Here we just give the definitions what we need about these $p$-local subgroups and we refer the reader to $[\mathbf{5 a 2}]$ for more details.

Definition 2.6. Let $(S, \mathcal{F}, \mathcal{L})$ be a $p$-local finite group and $\left(S_{0}, \mathcal{F}_{0}, \mathcal{L}_{0}\right)$ a $p$-local subgroup of $(S, \mathcal{F}, \mathcal{L})$. Set $\mathfrak{h y p}(\mathcal{F})=\left\langle g^{-1} \alpha(g) \mid g \in P \leqslant S, \alpha \in O^{p}\left(\operatorname{Aut}_{\mathcal{F}}(P)\right)\right\rangle \unlhd S$.

(a) We say that $\left(S_{0}, \mathcal{F}_{0}, \mathcal{L}_{0}\right)$ is a $p$-local subgroup of index a power of $p$ if $S_{0} \geqslant \mathfrak{h y p}(\mathcal{F})$ and, for every $P \leqslant S_{0}, O^{p}\left(\operatorname{Aut}_{\mathcal{F}}(P)\right) \leqslant \operatorname{Aut}_{\mathcal{F}_{0}}(P)$.

(b) We say that $\left(S_{0}, \mathcal{F}_{0}, \mathcal{L}_{0}\right)$ is a $p$-local subgroup of index prime to $p$ if $S_{0}=S$ and, for every $P \leqslant S, O^{p^{\prime}}\left(\operatorname{Aut}_{\mathcal{F}}(P)\right) \leqslant \operatorname{Aut}_{\mathcal{F}_{0}}(P)$.

Notice that $\mathfrak{h y p}(\mathcal{F})$ is denoted $O_{\mathcal{F}}^{p}(S)$ in [5a2, Definition 2.1]. These particular $p$-local subgroups satisfy the following properties.

Proposition 2.7 ([5a2, Proposition 3.8]). Let $(S, \mathcal{F}, \mathcal{L})$ be a p-local finite group and $\left(S_{0}, \mathcal{F}_{0}, \mathcal{L}_{0}\right)$ a p-local subgroup of $(S, \mathcal{F}, \mathcal{L})$

(a) If $\left(S_{0}, \mathcal{F}_{0}, \mathcal{L}_{0}\right)$ is of index a power of $p$, then $P \leqslant S_{0}$ is $\mathcal{F}_{0}$-quasicentric if, and only if, $P$ is $\mathcal{F}$-quasicentric.

(b) If $\left(S_{0}, \mathcal{F}_{0}, \mathcal{L}_{0}\right)$ is of index prime to $p$, then $P \leqslant S$ is $\mathcal{F}_{0}$-centric if, and only if, $P$ is $\mathcal{F}$-centric.

For an infinite group $G$, we denote by $O^{p^{\prime}}(G)$ the intersection of all normal subgroups in $G$ of finite index prime to $p$. For $\mathcal{F}$ a fusion system over a $p$-group $S$, let $O_{*}^{p^{\prime}}(\mathcal{F})$ be the fusion system generated by $O^{p^{\prime}}\left(\operatorname{Aut}_{\mathcal{F}}(P)\right)$ for all $P \leqslant S$ and define

$$
\operatorname{Out}_{\mathcal{F}}^{0}(S)=\left\langle\alpha \in \operatorname{Out}_{\mathcal{F}}(S)|\alpha|_{P} \in \operatorname{Hom}_{O_{*}^{p^{\prime}(\mathcal{F})}}(P, S) \text {, for some } P \leqslant S\right\rangle .
$$

Since $\operatorname{Aut}_{\mathcal{F}}(S)$ normalizes $O_{*}^{p^{\prime}}(\mathcal{F}), \operatorname{Out}_{\mathcal{F}}^{0}(S) \unlhd \operatorname{Out}_{\mathcal{F}}(S)$.

Proposition 2.8. Let $(S, \mathcal{F}, \mathcal{L})$ be a p-local finite group.

(a) $\mathcal{F}=\left\langle\operatorname{Aut}_{\mathcal{F}}(S), O_{*}^{p^{\prime}}(\mathcal{F})\right\rangle$.

(b) $\pi$ and the inclusion of $B \operatorname{Aut}_{\mathcal{F}}(S)$ in $\left|\mathcal{F}^{c}\right|$ induce isomorphisms,

$$
\theta: \pi_{\mathcal{L}} / O^{p^{\prime}}\left(\pi_{\mathcal{L}}\right) \stackrel{\cong}{\longrightarrow} \pi_{1}\left(\left|\mathcal{F}^{c}\right|\right) \stackrel{\cong}{\longrightarrow} \operatorname{Out}_{\mathcal{F}}(S) / O u t_{\mathcal{F}}^{0}(S)
$$

Proof. The point $(a)$ is proved in [5a2, Lemma 3.4]. For (b), the second isomorphism is given in [5a2, Proposition 5.2] and the first one in [5a2, Theorem 5.5] and the comment which follows.

According to Proposition 2.7, when dealing with $p$-local subgroups of index prime to $p$, we will work with centric linking systems. 
Theorem 2.9 ([5a2, Theorem 5.5]). Let $(S, \mathcal{F}, \mathcal{L})$ be a p-local finite group with $\mathcal{L}$ a centric linking system. For each subgroup $H \leqslant \operatorname{Out}_{\mathcal{F}}(S)$ containing $\operatorname{Out}_{\mathcal{F}}^{0}(S)$, there is a unique p-local finite subgroup $\left(S, \mathcal{F}_{H}, \mathcal{L}_{H}\right)$ of index prime to $p$ such that $\operatorname{Out}_{\mathcal{F}_{H}}(S)=$ $H$ and $\mathcal{L}_{H}=\pi^{-1}\left(\mathcal{F}_{H}^{c}\right)$.

Moreover, $\left|\mathcal{L}_{H}\right|$ is homotopy equivalent, via its inclusion in $|\mathcal{L}|$, to the covering space of $|\mathcal{L}|$ with fundamental group $\widetilde{H} \geqslant O^{p^{\prime}}\left(\pi_{\mathcal{L}}\right)$ such that $\theta\left(\widetilde{H} / O^{p^{\prime}}\left(\pi_{\mathcal{L}}\right)\right)=$ $H / O u t_{\mathcal{F}}^{0}(S)$ (where $\theta$ is the isomorphism given in Proposition 2.8(b)).

Thus, for a $p$-local finite group $(S, \mathcal{F}, \mathcal{L})$, with $\mathcal{L}$ a centric linking system, we can define the minimal $p$-local subgroup of index prime to $p,\left(S, O^{p^{\prime}}(\mathcal{F}), O^{p^{\prime}}(\mathcal{L})\right)$ corresponding to $\left(S, \mathcal{F}_{H}, \mathcal{L}_{H}\right)$ with $H=\operatorname{Out}_{\mathcal{F}}^{0}(S)$ in Theorem 2.9.

\subsection{Cohomology and stable elements}

The first result about stable elements is due to Cartan and Eilenberg [CE, Chap. XII, Theorem 10.1]. It also served as a guideline in the establishment of Theorem 1.1 by Broto, Levi and Oliver. Here we recall the definition of $\mathcal{F}^{c}$-stable elements in a context of twisted coefficients. We refer the reader to [Mo1] for more details. As in [Mo1], we will denote by $\omega: \mathcal{L} \rightarrow \pi_{\mathcal{L}}=\pi_{1}(|\mathcal{L}|, S)$ the functor which maps each object to the unique object in the target and sends each morphism $\varphi \in \operatorname{Mor}_{\mathcal{L}}(P, Q)$ to the class of the loop $\iota_{Q} \cdot \varphi \cdot \overline{\iota_{P}}$ where $\iota_{P}=\delta(1) \in \operatorname{Mor}_{\mathcal{L}}(P, S), \iota_{Q}=\delta(1) \in \operatorname{Mor}_{\mathcal{L}}(Q, S)$ and $\overline{\iota_{P}}$ is the edge $\iota_{P}$ followed in the opposite direction.

Let $(S, \mathcal{F}, \mathcal{L})$ be a $p$-local finite group. Recall first that $\delta: \mathcal{T}_{S}^{\mathrm{Ob}(\mathcal{L})}(S) \rightarrow \mathcal{L}$ induces an inclusion $\delta_{S}: B S \rightarrow|\mathcal{L}|$. In particular, it induces a natural map $S \rightarrow \pi_{\mathcal{L}}$ and thus, for every $\mathbb{Z}_{(p)}\left[\pi_{\mathcal{L}}\right]$-module $M$, we have a natural action of $S$, or any subgroup of $S$, on $M$. Now, let $M$ be a $\mathbb{Z}_{(p)}\left[\pi_{\mathcal{L}}\right]$-module, the group cohomology bifunctor $H^{*}(-,-)$ induces a functor

$$
H^{*}(-, M): \mathcal{F}^{c} \longrightarrow \mathbb{Z}_{(p)}-\operatorname{Mod}
$$

(a priori, $H^{*}(g, M)$ is defined for $g \in \operatorname{Mor}(\mathcal{L})$ but [Mo1, Proposition 2.2] proves that $H^{*}(-, M)$ is well defined on $\left.\mathcal{F}^{c}\right)$.

Definition 2.10. Let $(S, \mathcal{F}, \mathcal{L})$ be a $p$-local finite group. An element $x \in H^{*}(S, M)$ is called $\mathcal{F}$-centric stable, or $\mathcal{F}^{c}$-stable, if for all $P \in \operatorname{Ob}\left(\mathcal{F}^{c}\right)$ and all $\varphi \in \operatorname{Hom}_{\mathcal{F}}(P, S)$,

$$
\varphi^{*}(x)=\operatorname{Res}_{P}^{S}(x) .
$$

We denote by $H^{*}\left(\mathcal{F}^{c}, M\right) \subseteq H^{*}(S, M)$ the submodule of all $\mathcal{F}^{c}$-stable elements.

Notice that

$$
H^{*}\left(\mathcal{F}^{c}, M\right)=\lim _{\overleftarrow{\mathcal{F}^{c}}} H^{*}(-, M)=\lim _{\overleftarrow{\mathcal{L}}} H^{*}(-, M)
$$

where the last equality holds if $\mathcal{L}$ is a centric linking system.

\section{Constrained fusion systems}

Let $(S, \mathcal{F}, \mathcal{L})$ be a $p$-local finite group. Here, we assume that $\mathcal{F}$ is a constrained fusion system. 
Definition 3.1. Let $\mathcal{F}$ be a fusion system over a $p$-group $S$. A $\operatorname{subgroup} Q \leqslant S$ is normal in $\mathcal{F}$ if

(i) $Q \unlhd S$, and

(ii) for all $P, R \leqslant S$ and every $\varphi \in \operatorname{Hom}_{\mathcal{F}}(P, R), \varphi$ extends to a morphism $\bar{\varphi} \in$ $\operatorname{Hom}_{\mathcal{F}}(P Q, R Q)$ such that $\bar{\varphi}(Q)=Q$.

We write $O_{p}(\mathcal{F})$ for the maximal subgroup of $S$ which is normal in $\mathcal{F}$. We say that $\mathcal{F}$ is constrained if $O_{p}(\mathcal{F})$ is $\mathcal{F}$-centric.

An important and classical result about constrained fusion systems is the following.

Proposition 3.2 ([5a1, Proposition 4.3]). Let $(S, \mathcal{F}, \mathcal{L})$ be a p-local finite group with $\mathcal{L}$ a centric linking system. If $\mathcal{F}$ is constrained, there exists a finite group $G$ such that

(a) $S$ is a Sylow p-subgroup of $G$,

(b) $C_{G}\left(O_{p}(G)\right) \leqslant O_{p}(G)$,

(c) $\mathcal{F}_{S}(G)=\mathcal{F}$.

Moreover, $G \cong \operatorname{Aut}_{\mathcal{L}}\left(O_{p}(\mathcal{F})\right)$ and $\mathcal{L} \cong \mathcal{L}_{S}^{c}(G)$.

This group $G$ is called a model of $\mathcal{F}$ and it is unique in a precise way (see $[\mathbf{A K O}$, Theorem III.5.10]). This model can also be recovered from the homotopy type of the geometric realization of a linking system associated to $\mathcal{F}$.

Lemma 3.3. Let $(S, \mathcal{F}, \mathcal{L})$ be a p-local finite group with $\mathcal{L}$ a centric linking system. If $\mathcal{F}$ is constrained, then $|\mathcal{L}|$ is a classifying space of a model $G$ of $\mathcal{F}$.

Proof. By Proposition 3.2, we can assume that $\mathcal{L}=\mathcal{L}_{S}^{c}(G)$. Set

$$
\mathcal{H}=\left\{P \in \mathrm{Ob}(\mathcal{L}) \mid P \geqslant O_{p}(G)\right\}
$$

and let $\mathcal{L}^{\mathcal{H}}$ be the full subcategory of $\mathcal{L}$ with set of objects $\mathcal{H}$. By [5a1, Proposition 1.6], $\mathcal{H}$ contains all $\mathcal{F}$-centric and $\mathcal{F}$-radical subgroups. Thus, by Proposition 2.3, $\mathcal{L}^{\mathcal{H}}$ is a linking system associated to $\mathcal{F}$ and, by Theorem $2.5,\left|\mathcal{L}^{\mathcal{H}}\right| \cong|\mathcal{L}|$.

It remains to prove that $\left|\mathcal{L}^{\mathcal{H}}\right| \cong B G$. For that purpose, consider the following functor:

$$
\begin{aligned}
F: \quad \mathcal{L}^{\mathcal{H}} & \longmapsto \mathcal{L}^{\left\{O_{p}(G)\right\},} \\
P \in \mathcal{L}^{\mathcal{H}} & \longmapsto O_{p}(G), \\
g \in T_{G}(P, Q) & \longmapsto g \in N_{G}\left(O_{p}(G)\right)=G .
\end{aligned}
$$

It gives us a retraction by deformation of $\left|\mathcal{L}^{\mathcal{H}}\right|$ on the geometric realization of the full subcategory of $\mathcal{L}$ with unique object $O_{p}(G) \leqslant S$. As $\operatorname{Aut}_{\mathcal{L}}\left(O_{p}(G)\right)=N_{G}\left(O_{p}(G)\right)=$ $G$, this last category is $\mathcal{B}(G)$. In particular, its geometric realization is a classifying space of $G$.

Proposition 3.4. Let $G$ be a finite group and $S$ a Sylow p-subgroup of $G$. If we have $C_{G}\left(O_{p}(G)\right) \leqslant O_{p}(G)$, then, for every $\mathbb{Z}_{(p)}[G]$-module $M$, the inclusion of $S$ in $G$ induces a natural isomorphism

$$
H^{*}(G, M) \cong H^{*}\left(\mathcal{F}_{S}^{c}(G), M\right)
$$


Proof. Let $(S, \mathcal{F}, \mathcal{L})=\left(S, \mathcal{F}_{S}(G), \mathcal{L}_{S}^{c}(G)\right)$. By assumption, $\mathcal{F}_{S}(G)$ is constrained and $G$ is a model of $\mathcal{F}_{S}(G)$. From Cartan-Eilenberg Theorem, we know that

$$
\operatorname{Res}_{S}^{G}: H^{*}(G, M) \longrightarrow H^{*}(S, M)
$$

is injective and that $\operatorname{Im}\left(\operatorname{Res}_{S}^{G}\right)=\lim _{\mathcal{T}_{S}(G)} H^{*}(-, M)$. Moreover,

$$
H^{*}\left(\mathcal{F}^{c}, M\right)=\lim _{\mathcal{F}^{c}} H^{*}(-, M)=\lim _{\overleftarrow{L}} H^{*}(-, M)=\lim _{\mathcal{T}_{S}^{c}(G)} H^{*}(-, M) \geqslant \lim _{\mathcal{T}_{S}(G)} H^{*}(-, M) .
$$

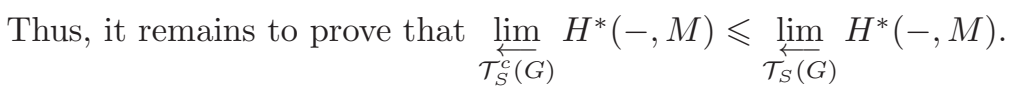

Let then $x \in H^{*}\left(\mathcal{F}^{c}, M\right)=\lim _{\mathcal{T}_{S}^{c}(G)} H^{*}(-, M)$. For $P \leqslant S$ and $g \in N_{G}(P, S)$ we have, in $\mathcal{T}_{S}(G)$, the following commutative diagram:

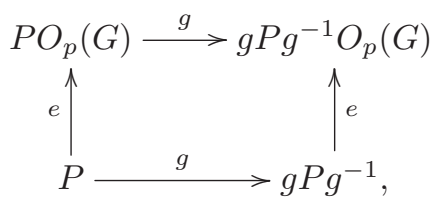

where $e$ is the trivial element of $G$. Hence, as the top part of the diagram is in $T_{S}^{c}(G)$ and $x \in \lim _{\mathcal{T}_{S}^{c}(G)} H^{*}(-, M)$,

$$
\begin{aligned}
c_{g}^{*} \circ \operatorname{Res}_{g P g^{-1}}^{S}(x) & =\operatorname{Res}_{P}^{P O^{p}(G)} \circ c_{g}^{*} \circ \operatorname{Res}_{g P g^{-1} O_{p}(G)}^{S}(x) \\
& =\operatorname{Res}_{P}^{P O^{p}(G)} \circ \operatorname{Res}_{P O_{p}(G)}^{S}(x) \\
& =\operatorname{Res}_{P}^{S}(x) .
\end{aligned}
$$

Thus $x \in \underset{\mathcal{T}_{S}(G)}{\lim _{(}} H^{*}(-, M)$ and this complete the proof.

Corollary 3.5. Let $(S, \mathcal{F}, \mathcal{L})$ be a p-local finite group. If $\mathcal{F}$ is constrained and $M$ is $a \mathbb{Z}_{(p)}\left[\pi_{\mathcal{L}}\right]$-module, then $\delta_{S}$ induces a natural isomorphism,

$$
H^{*}(|\mathcal{L}|, M) \cong H^{*}\left(\mathcal{F}^{c}, M\right)
$$

\section{Actions factoring through a $p^{\prime}$-group}

In this section, for each $p$-local finite group $(S, \mathcal{F}, \mathcal{L})$ we will assume that $\mathcal{L}$ is a centric linking system.

Lemma 4.1. Let $(S, \mathcal{F}, \mathcal{L})$ be a p-local finite group and $\left(S, O^{p^{\prime}}(\mathcal{F}), O^{p^{\prime}}(\mathcal{L})\right)$ its minimal p-local subgroup of index prime to $p$. If $M$ is a $\mathbb{Z}_{(p)}\left[\pi_{\mathcal{L}}\right]$-module, then the inclusion $O^{p^{\prime}}(\mathcal{L}) \subseteq \mathcal{L}$ induces the following isomorphism,

$$
H^{*}(|\mathcal{L}|, M) \cong H^{*}\left(\left|O^{p^{\prime}}(\mathcal{L})\right|, M\right)^{\pi_{\mathcal{L}} / O^{p^{\prime}}\left(\pi_{\mathcal{L}}\right)} .
$$


Proof. By Theorem 2.9, $\left|O^{p^{\prime}}(\mathcal{L})\right|$ is, up to homotopy, a covering space of $|\mathcal{L}|$ with fundamental group $O^{p^{\prime}}\left(\pi_{\mathcal{L}}\right) \unlhd \pi_{\mathcal{L}}$. It gives us a fibration sequence

$$
\left|O^{p^{\prime}}(\mathcal{L})\right| \rightarrow|\mathcal{L}| \rightarrow B\left(\pi_{\mathcal{L}} / O^{p^{\prime}}\left(\pi_{\mathcal{L}}\right)\right) .
$$

Consider then the Serre spectral sequence associated

$$
H^{s+t}(|\mathcal{L}|, M) \Leftarrow H^{s}\left(\pi_{\mathcal{L}} / O^{p^{\prime}}\left(\pi_{\mathcal{L}}\right), H^{t}\left(\left|O^{p^{\prime}}(\mathcal{L})\right|, M\right)\right) .
$$

$M$ is a $\mathbb{Z}_{(p)}$-module, thus $H^{q}\left(\left|O^{p^{\prime}}(\mathcal{L})\right|, M\right)$ is also a $\mathbb{Z}_{(p)}$-module. As $\pi_{\mathcal{L}} / O^{p^{\prime}}\left(\pi_{\mathcal{L}}\right)$ is a $p^{\prime}$-group, the $E_{2}$-page is concentrated in the first column with terms

$$
H^{t}\left(\left|O^{p^{\prime}}(\mathcal{L})\right|, M\right)^{\pi_{\mathcal{L}} / O^{p^{\prime}}\left(\pi_{\mathcal{L}}\right)} .
$$

Thus the spectral sequence collapses on the $E_{2}$-page and the lemma follows.

Lemma 4.2. Let $(S, \mathcal{F}, \mathcal{L})$ be a p-local finite group and $\left(S, O^{p^{\prime}}(\mathcal{F}), O^{p^{\prime}}(\mathcal{L})\right)$ its minimal p-local subgroup of index prime to $p$. If $M$ is a $\mathbb{Z}_{(p)}\left[\pi_{\mathcal{L}}\right]$-module, then

$$
H^{*}\left(\mathcal{F}^{c}, M\right)=H^{*}\left(O^{p^{\prime}}(\mathcal{F})^{c}, M\right)^{A u t_{\mathcal{F}}(S) / A u t_{O p^{\prime}(\mathcal{F})}(S)} .
$$

Proof. Notice first that, by Proposition 2.7, $\mathrm{Ob}\left(O^{p^{\prime}}(\mathcal{F})^{c}\right)=\mathrm{Ob}\left(\mathcal{F}^{c}\right)$. Hence, we are working with the same underlying set of objects. Thus, by definition, $H^{*}\left(\mathcal{F}^{c}, M\right) \subseteq$ $H^{*}\left(O^{p^{\prime}}(\mathcal{F})^{c}, M\right)^{\operatorname{Aut}_{\mathcal{F}}(S) / \operatorname{Aut}_{O^{p^{\prime}}(\mathcal{F})}(S)}$. On the other hand, by Proposition 2.8, we have $\mathcal{F}=\left\langle O^{p^{\prime}}(\mathcal{F}), \operatorname{Aut}_{\mathcal{F}}(S)\right\rangle$ which gives the converse inclusion.

Theorem 4.3. Let $(S, \mathcal{F}, \mathcal{L})$ be a p-local finite group and $\left(S, O^{p^{\prime}}(\mathcal{F}), O^{p^{\prime}}(\mathcal{L})\right)$ its minimal p-local subgroup of index prime to $p$. If $M$ is a $\mathbb{Z}_{(p)}\left[\pi_{\mathcal{L}}\right]$-module and if the inclusion $\delta_{S}$ induces an isomorphism

$$
H^{*}\left(\left|O^{p^{\prime}}(\mathcal{L})\right|, M\right) \cong H^{*}\left(O^{p^{\prime}}(\mathcal{F})^{c}, M\right),
$$

then $\delta_{S}$ induces an isomorphism

$$
H^{*}(|\mathcal{L}|, M) \cong H^{*}\left(\mathcal{F}^{c}, M\right) .
$$

Proof. Recall that, by Theorem $2.9, \pi_{1}\left(\left|O^{p^{\prime}}(\mathcal{L})\right|\right)=O^{p^{\prime}}\left(\pi_{\mathcal{L}}\right)$. Then we have the following commutative diagram:

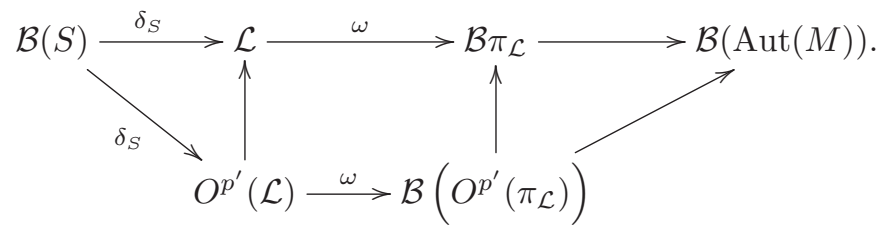

Moreover, by Proposition 2.8 and Theorem 2.9, the projection $\pi: \mathcal{L} \longrightarrow \mathcal{F}$ induces an isomorphism

$$
\pi_{\mathcal{L}} / O^{p^{\prime}}\left(\pi_{\mathcal{L}}\right) \cong \pi_{1}\left(\left|\mathcal{F}^{c}\right|\right) \cong \operatorname{Aut}_{\mathcal{F}}(S) / \operatorname{Aut}_{O^{p^{\prime}}(\mathcal{F})}(S) .
$$


Then, by the two previous lemmas, we obtain

$$
\begin{aligned}
H^{*}(|\mathcal{L}|, M) & \cong H^{*}\left(\left|O^{p^{\prime}}(\mathcal{L})\right|, M\right)^{\pi_{\mathcal{L}} / O^{p^{\prime}}\left(\pi_{\mathcal{L}}\right)} \\
& \cong\left(\lim _{O_{\mathcal{p}^{\prime}}(\mathcal{F})^{c}} H^{*}(-, M)\right)^{\operatorname{Aut}_{\mathcal{F}}(S) / \operatorname{Aut}_{O p^{\prime}(\mathcal{F})}(S)} \\
& \cong H^{*}\left(\mathcal{F}^{c}, M\right) .
\end{aligned}
$$

For the second isomorphism, we have to be careful with respect to the action of $\pi_{\mathcal{L}}$ on the left side of the isomorphism and $\operatorname{Aut}_{\mathcal{F}}(S)$ on the right side. In fact, here, by Definition 2.10 of $\mathcal{F}^{c}$-stable elements, we can see it on the chain level. The map $\delta_{S}^{*}: H^{*}\left(\left|O^{p^{\prime}}(\mathcal{L})\right|, M\right) \longrightarrow H^{*}(S, M)$, induced by $\delta_{S}: B S \longrightarrow\left|O^{p^{\prime}}(\mathcal{L})\right|$, gives on the chain level,

$$
\begin{gathered}
\operatorname{Hom}_{\mathbb{Z}_{(p)}[S]}\left(C_{*}\left(\left|\widehat{O^{p^{\prime}}(\mathcal{L})}\right|\right), M\right) \longrightarrow \operatorname{Hom}_{\mathbb{Z}_{(p)}\left[\pi_{O^{p^{\prime}}(\mathcal{L})}\right]}\left(C_{*}(|\mathcal{E}(S)|), M\right) \\
f \longmapsto \\
\left.\longrightarrow f\right|_{C_{*}(|\mathcal{E}(S)|)},
\end{gathered}
$$

where $\mathcal{E}(S)$ is defined as the category with set of object $S$ and for each $\left(s, s^{\prime}\right) \in S$, $\operatorname{Mor}_{\mathcal{E}(S)}\left(s, s^{\prime}\right)=\left\{\varphi_{s, s^{\prime}}\right\}$ (in particular, $|\mathcal{E}(S)|$ is a universal covering space of $B S$ ). Then, for $\varphi \in \operatorname{Aut}_{S}(\mathcal{F})$, if we choose a lift $\widetilde{\varphi} \in \operatorname{Aut}_{\mathcal{L}}(S), \varphi$ acts on the left side by

$$
f \longmapsto \omega\left(\widetilde{\varphi}^{-1}\right) f \omega(\widetilde{\varphi})
$$

and on the right side by,

$$
f \longmapsto \omega(\widetilde{\varphi})^{-1} f \circ \varphi^{*} .
$$

Finally, the action of $\varphi$ on $\mathcal{E}(S)$ corresponds to the action of $\omega(\widetilde{\varphi})$ on $|\mathcal{E}(S)|$ (indeed, a lift of $\omega(\widetilde{\varphi})$ in $\mid \widehat{O^{p^{\prime}}(\mathcal{L}) \mid}$ joins every vertex $s \in S$ of $|\mathcal{E}(S)|$ to the vertex $\varphi(s)$ and similarly for higher simplices). Hence, the two actions coincide.

Corollary 4.4. Let $(S, \mathcal{F}, \mathcal{L})$ be a p-local finite group and $M$ be a $\mathbb{Z}_{(p)}\left[\pi_{\mathcal{L}}\right]$-module. If the action of $\pi_{\mathcal{L}}$ on $M$ factors through a $p^{\prime}$-group then $\delta_{S}$ induces an isomorphism,

$$
H^{*}(|\mathcal{L}|, M) \cong H^{*}\left(\mathcal{F}^{c}, M\right) .
$$

Proof. By Theorem 4.3, it is enough to prove that $\delta_{S}$ induces an isomorphism

$$
H^{*}\left(\left|O^{p^{\prime}}(\mathcal{L})\right|, M\right) \cong H^{*}\left(O^{p^{\prime}}(\mathcal{F})^{c}, M\right) .
$$

But, as the action on $M$ factor through a $p^{\prime}$-group, $\pi_{1}\left(\left|O^{p^{\prime}}(\mathcal{L})\right|\right)=O^{p^{\prime}}\left(\pi_{\mathcal{L}}\right)$ acts trivially on $M$ and Theorem 1.1 gives the wanted isomorphism.

We already know, from a previous article [Mo1, Theorem 4.3] that, if $M$ is a finite $\mathbb{Z}_{(p)}\left[\pi_{\mathcal{L}}\right]$-module and the action of $\pi_{\mathcal{L}}$ on $M$ factor through a $p$-group, then $\delta_{S}$ induces an isomorphism

$$
H^{*}(|\mathcal{L}|, M) \cong H^{*}\left(\mathcal{F}^{c}, M\right)
$$

(it is a direct corollary of [Mo1, Theorem 4.3] because, any action of a $p$-group on an abelian $p$-group is nilpotent). Hence, with the same arguments, we get another corollary of Theorem 4.3 . 
Corollary 4.5. Let $(S, \mathcal{F}, \mathcal{L})$ be a p-local finite group and $M$ be a finite $\mathbb{Z}_{(p)}\left[\pi_{\mathcal{L}}\right]$ module. If the action of $\pi_{\mathcal{L}}$ on $M$ factors through an extension of a normal p-group by a $p^{\prime}$-group then $\delta_{S}$ induces an isomorphism,

$$
H^{*}(|\mathcal{L}|, M) \cong H^{*}\left(\mathcal{F}^{c}, M\right) .
$$

\section{Realizable fusion systems and actions factoring through a $p$-solvable group}

Consider here a finite group $G, S$ a Sylow $p$-subgroup of $G$ and let $(S, \mathcal{F}, \mathcal{L})$ be the associated $p$-local finite group with $\mathcal{L}=\mathcal{L}_{S}^{c}(G)$. Set $\mathcal{T}=\mathcal{T}_{S}^{c}(G)$ be the centric transporter category of $G, \mathcal{L}^{q}=\mathcal{L}_{S}^{q}(G)$ be the quasicentric linking system associated to $G$ and $\mathcal{T}^{q}=\mathcal{T}_{S}^{q}(G)$ be the associated quasicentric transporter category. We also write $\pi_{\mathcal{T}}=\pi_{1}(|\mathcal{T}|)$.

We have a functor

$$
\rho: \mathcal{T}_{S}(G) \longrightarrow \mathcal{B}(G)
$$

which sends each object in the source to the unique object $o_{G}$ in the target and sends, for every $P, Q \leqslant S, g \in T_{G}(P, Q)$ to $g \in G=\operatorname{Mor}_{\mathcal{B}}(G)\left(o_{G}\right)$. As $|\mathcal{B}(G)|=B G$, this induces a homomorphism

$$
\rho_{*}: \pi_{\mathcal{T}} \longrightarrow G .
$$

Here for $M$ a $\mathbb{Z}_{(p)}\left[\pi_{\mathcal{L}}\right]$-module, with action $\varphi: \pi_{\mathcal{L}} \rightarrow \operatorname{Aut}(M)$ we will suppose that we have the following commutative diagram for some homomorphism $\bar{\varphi}: G \rightarrow$ $\operatorname{Aut}(M)$ :

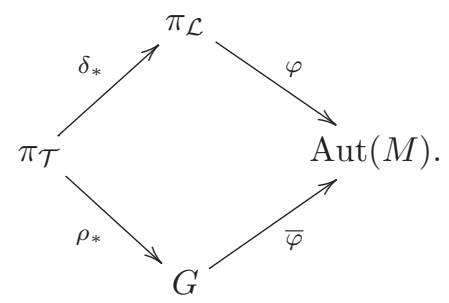

Then, we can compare the cohomology of $|\mathcal{L}|$ and the cohomology of $G$ when the action factors through a $p$-solvable group. The main ingredients that we will use are $p$-local subgroups of index a power of $p$ or prime to $p$.

The following lemma allows us to compare $H^{*}(|\mathcal{L}|, M)$ and $H^{*}(|\mathcal{T}|, M)$.

Lemma 5.1. Let $G$ be a finite group and $(S, \mathcal{F}, \mathcal{L})$ be an associated p-local finite group. Let $\mathcal{T}=\mathcal{T}_{S}{ }^{O b(\mathcal{L})}(G) \subseteq \mathcal{T}^{q}$ be the transporter category associated to $G$ with set of objects $\operatorname{Ob}(\mathcal{L})$. If $M$ is a $\mathbb{Z}_{(p)}\left[\pi_{\mathcal{L}}\right]$-module, then the canonical functor $\delta: \mathcal{T} \rightarrow \mathcal{L}$ induces a natural isomorphism $H^{*}(|\mathcal{T}|, M) \cong H^{*}(|\mathcal{L}|, M)$.

Proof. This is a consequence of [BLO1, Lemma 1.3] with $\mathcal{C}=\mathcal{T}, \mathcal{C}^{\prime}=\mathcal{L}$ and the functor $T: \mathcal{L}^{\text {op }} \rightarrow \mathbb{Z}_{(p)}$-Mod which sends each object to $M$, and each morphism to its 
action on $M$. Then $\delta$ induces a natural isomorphism ${\underset{\mathcal{T}}{\lim }}^{*}(M) \cong{\underset{\mathcal{L}}{\mathcal{L}}}^{\lim } *(M)$. Then

$$
H^{*}(|\mathcal{T}|, M)={\underset{\leftarrow}{\mathcal{T}}}_{\lim } *(M) \cong{\underset{\leftarrow}{\mathcal{L}}}_{\lim } *(M)=H^{*}(|\mathcal{L}|, M)
$$

where the first and last equality is just an interpretation in terms of functor cohomology and can be found in [LR, Proposition 3.9].

Theorem 5.2. Let $G$ be a finite group, $S$ a Sylow p-subgroup of $G, \mathcal{L}=\mathcal{L}_{S}^{c}(G)$ and $\mathcal{T}=\mathcal{T}_{S}^{c}(G)$. Let $M$ be a $\mathbb{Z}_{(p)}\left[\pi_{\mathcal{L}}\right]$-module and assume that we have the following commutative diagram:

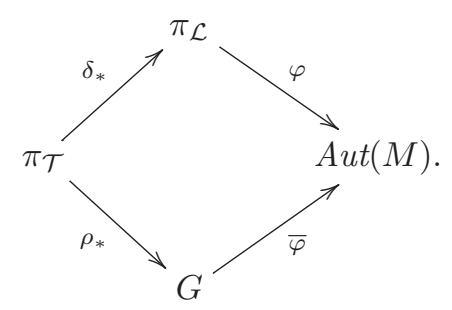

If $\rho_{*}$ is surjective and $\Gamma=\operatorname{Im}(\varphi)=\operatorname{Im}(\bar{\varphi})$ is p-solvable, then $\delta$ and $\rho$ induce natural isomorphisms

$$
H^{*}(|\mathcal{L}|, M) \cong H^{*}(|\mathcal{T}|, M) \cong H^{*}(G, M)
$$

Proof. By Lemma 5.1, we just have to show that $\rho$ induces a natural isomorphism $H^{*}(|\mathcal{T}|, M) \cong H^{*}(G, M)$. We prove this by induction on the minimal number $n$ of extensions by $p$-groups or $p^{\prime}$-groups we need to obtain $\Gamma$.

If $n=0, \Gamma=1$ and the action of $\pi_{\mathcal{T}}$ on $M$ is trivial, then it follows from [OV 1 , Proposition 4.5]. Assume that, if $\Gamma$ is obtained by $n$ extensions, the result is true and suppose that $\Gamma$ is obtained with $n+1$ extensions. Consider then the last one

$$
0 \rightarrow \Gamma_{n} \rightarrow \Gamma \rightarrow Q \rightarrow 0
$$

Denote $H=\bar{\varphi}_{*}^{-1}\left(\Gamma_{n}\right)$. Thus $\left(T, \mathcal{F}_{H}, \mathcal{L}_{H}\right)=\left(S \cap H, \mathcal{F}_{S \cap H}(H), \mathcal{L}_{S \cap H}^{c}(H)\right)$ is a $p$-local subgroup of $(S, \mathcal{F}, \mathcal{L})$ of index a power of $p$ or prime to $p$.

If $Q$ is a $p^{\prime}$-group. In that case, $\left(T, \mathcal{F}_{H}, \mathcal{L}_{H}\right)$ is a $p$-local finite subgroup of index prime to $p$ (defined in Definition 2.6). Then $\operatorname{Ob}\left(\mathcal{F}^{c}\right)=\operatorname{Ob}\left(\mathcal{F}_{H}^{c}\right), \mathcal{T}_{H}=\mathcal{T}_{S \cap H}^{c}(H) \subset \mathcal{T}$ and, by [OV1, Proposition 4.1(d)], this inclusion of categories induces, up to homotopy, a covering space of $|\mathcal{T}|$ with covering group $G / H=Q$. We then have the following commutative diagram with exact rows (here, $\longrightarrow$ means onto):

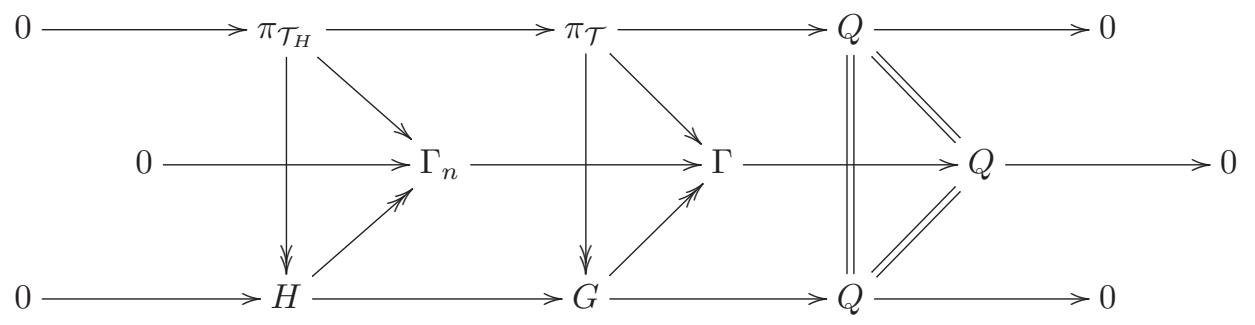


and the following fibration sequences

$$
\begin{aligned}
& \left|\mathcal{T}_{H}\right| \longrightarrow|\mathcal{T}| \longrightarrow B Q \\
& B H \longrightarrow B G \longrightarrow B Q .
\end{aligned}
$$

Moreover, $\rho$ induces a morphism of fibration sequences between these two.

If $Q$ is a p-group. In that case, we have to be more careful on the collection of subgroups of $S$ we are working with. As in the case when $Q$ is a $p^{\prime}$-group we want to apply [OV1, Proposition 4.1(d)]. This forces us to use the following collection. Let

$$
\mathcal{H}=\left\{P \in \mathrm{Ob}\left(\mathcal{F}^{q}\right) \mid P \cap T \in \mathrm{Ob}\left(\mathcal{F}_{H}^{q}\right)\right\} .
$$

Since $H \unlhd G$, no element of $T=S \cap H$ is $G$-conjugate to any element of $S \backslash T$. Thus, by [5a2, Lemma 3.5], for every $P \in \mathrm{Ob}\left(\mathcal{F}^{c r}\right), P \cap T \in \mathrm{Ob}\left(\mathcal{F}_{H}^{c}\right) \subseteq \operatorname{Ob}\left(\mathcal{F}_{H}^{q}\right)$. In particular, $\mathrm{Ob}\left(\mathcal{F}^{c r}\right) \subseteq \mathcal{H} \subseteq \mathrm{Ob}\left(\mathcal{F}^{q}\right)$. Hence if $\mathcal{L}^{\mathcal{H}} \subseteq \mathcal{L}^{q}$ is the full subcategory of $\mathcal{L}^{q}$ with set of objects $\mathcal{H}$, by Proposition 2.3(b), $\mathcal{L}^{\mathcal{H}}$ defines a linking system associated to $\mathcal{F}$. On the level of transporter systems, the inclusions $\mathcal{T} \subseteq \mathcal{T}^{q} \supseteq \mathcal{T}^{\mathcal{H}}$ induce natural isomorphisms $H^{*}\left(\left|\mathcal{T}^{\mathcal{H}}\right|, M\right) \simeq H^{*}\left(\left|\mathcal{T}^{q}\right|, M\right) \simeq H^{*}(|\mathcal{T}|, M)$. Indeed, we have the following commutative diagram:

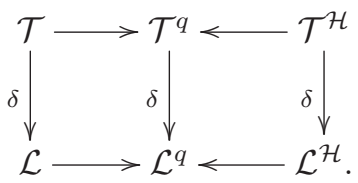

The vertical arrows induce isomorphisms in cohomology by Lemma 5.1 and the lower horizontal one induces an isomorphism since, by Theorem 2.5, the inclusions of categories $\mathcal{L} \subseteq \mathcal{L}^{q} \supseteq \mathcal{L}^{\mathcal{H}}$ induces $|\mathcal{L}| \simeq\left|\mathcal{L}^{q}\right| \simeq\left|\mathcal{L}^{\mathcal{H}}\right|$. Hence the upper arrows induce isomorphisms $H^{*}\left(\left|\mathcal{T}^{\mathcal{H}}\right|, M\right) \simeq H^{*}\left(\left|\mathcal{T}^{q}\right|, M\right) \simeq H^{*}(|\mathcal{T}|, M)$. Finally, by Proposition 2.7, $P \in \mathrm{Ob}\left(\mathcal{F}_{H}^{q}\right)$ if and only if $P \leqslant T$ and $P \in \mathcal{H}$. In particular, $\mathcal{T}_{H}^{q} \subseteq \mathcal{T}^{\mathcal{H}}$. Thus we can assume for this part that $\mathcal{T}=\mathcal{T}^{\mathcal{H}}$ and $\mathcal{T}_{H}=\mathcal{T}_{H}^{q}$.

We have $\mathcal{T}_{H} \subseteq \mathcal{T}$ is a transporter system associated to $\mathcal{F}_{H}$ and, by definition of $\mathcal{H}$, the hypotheses of [OV1, Proposition 4.1(d)], are satisfied. Thus this inclusion induces a covering space of $|\mathcal{T}|$ with covering group $G / H=Q$. Therefore, we have the following diagram with exact rows

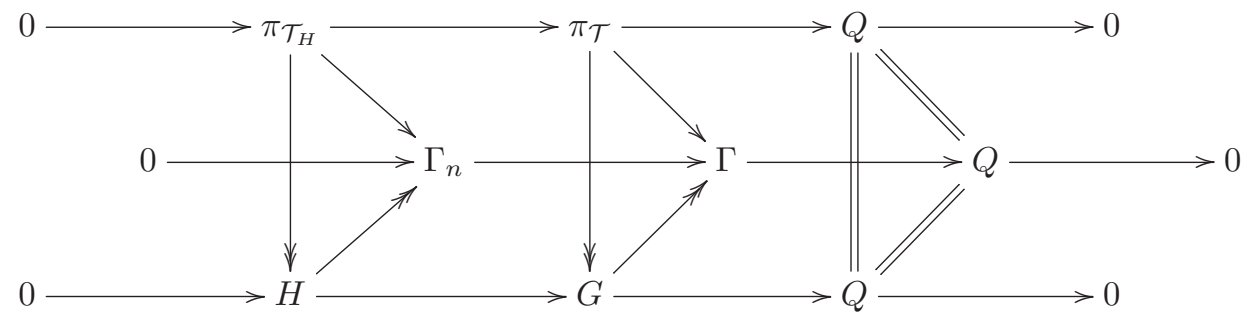

and the following fibration sequences

$$
\begin{aligned}
& \left|\mathcal{T}_{H}\right| \longrightarrow|\mathcal{T}| \longrightarrow B Q, \\
& B H \longrightarrow B G \longrightarrow B Q .
\end{aligned}
$$

Moreover, $\rho$ induces a morphism of fibration sequences between these two. 
Hence, in both cases, we have the following Serre spectral sequences

$$
\begin{aligned}
H^{s+t}(|\mathcal{T}|, M) & \Leftarrow H^{s}\left(Q, H^{t}\left(\left|\mathcal{T}_{H}\right|, M\right)\right), \\
H^{s+t}(G, M) & \Leftarrow H^{s}\left(Q, H^{t}(H, M)\right),
\end{aligned}
$$

and $\rho$ induces a morphism $\rho^{*}$ of spectral sequences between these two. By induction, $\rho^{*}$ gives an isomorphism on the $E_{2}$ page and then induces an isomorphism of spectral sequences. In particular, $\rho$ induces a natural isomorphism

$$
H^{*}(|\mathcal{T}|, M) \cong H^{*}(G, M)
$$

The result follows by induction.

Assume the hypotheses of Theorem 5.2. It remains to compare $H^{*}(G, M)$ with the $\mathcal{F}^{c}$-stable elements. This is also not obvious and they are not isomorphic in all cases. On one hand, by Cartan-Eilenberg Theorem, we have $H^{*}(G, M) \cong \lim _{\mathcal{T}_{S}^{\leftarrow}(G)} H^{*}(-, M)$.

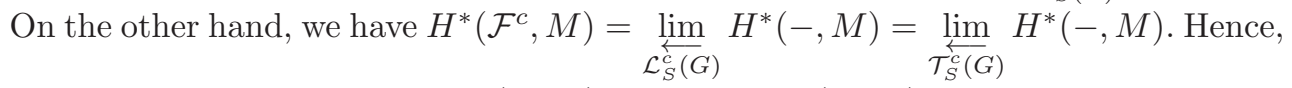
it remains to compare $\lim _{\mathcal{T}_{S}^{\leftarrow}(G)} H^{*}(-, M)$ and $\lim _{\mathcal{T}_{S}^{c}(G)} H^{*}(-, M)$. For that we can use a result of Grodal $[\mathbf{G r}]$.

Definition 5.3. let $G$ be a finite group, $S \in \operatorname{Syl}_{p}(G)$ and $M$ be a $\mathbb{Z}_{(p)}[G]$-module. Let $K$ be the kernel of $G \rightarrow \operatorname{Aut}(M)$. A subgroup $P \leqslant S$ is called $M$-essential if

(i) the poset of non-trivial $p$-subgroup of $N_{G}(P) / P$ is empty or disconnected,

(ii) $Z(P) \cap K \in \operatorname{Syl}_{p}\left(C_{G}(P) \cap K\right)$,

(iii) $O_{p}\left(N_{G}(P) /\left(P\left(C_{G}(P) \cap K\right)\right)\right)=1$.

The property (ii) looks like the definition of $p$-centric and (iii) looks like the definition of $\mathcal{F}$-radical. For the property (i), if $P$ is $\mathcal{F}$-centric and fully normalized in $\mathcal{F}$, it is equivalent to $P=S$ or $P$ is $\mathcal{F}$-essential [AKO, Definition I.3.2].

Theorem 5.4 ([Gr, Corollary 10.4]). Let $G$ be a finite group, $S$ a Sylow p-subgroup of $G$ and $M$ a $\mathbb{Z}_{(p)}[G]$-module.

Let $\mathcal{H}$ be a family of subgroup of $S$ containing $S$ and all the subgroups which are $M$-essential.

Then, the inclusion of $S$ in $G$ induce a natural isomorphism,

$$
H^{*}(G, M) \cong \lim _{\mathcal{T}_{S}^{\mathcal{H}}(G)} H^{*}(-, M)
$$

From this theorem and Theorem 5.2, we get the following corollary.

Corollary 5.5. Let $G$ be a finite group, $S$ a Sylow p-subgroup of $G$ and $(S, \mathcal{F}, \mathcal{L})$ the associated p-local finite group. Let $M$ be a $\mathbb{Z}_{(p)}\left[\pi_{\mathcal{L}}\right]$-module and assume that we have 
the following commutative diagram:

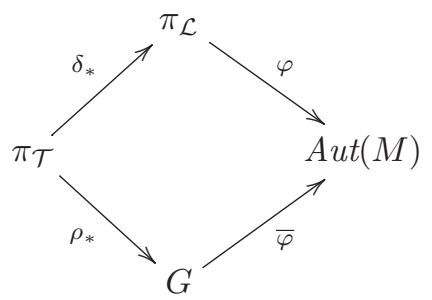

that $\rho_{*}$ is surjective and that $\Gamma:=\operatorname{Im}(\varphi)=\operatorname{Im}(\bar{\varphi})$. If $\Gamma$ is $p$-solvable and all the $M$-essential subgroups of $S$ are $p$-centric, then $\delta$ and $\rho$ induce natural isomorphisms,

$$
H^{*}(|\mathcal{L}|, M) \cong H^{*}(G, M) \cong H^{*}\left(\mathcal{F}^{c}, M\right) .
$$

We also conjecture that it can be generalized to any abstract $p$-local finite group and any $\mathbb{Z}_{(p)}\left[\pi_{\mathcal{L}}\right]$-module with a $p$-solvable action.

Conjecture 5.6. Let $(S, \mathcal{F}, \mathcal{L})$ be a p-local finite group and let $M$ be a $\mathbb{Z}_{(p)}\left[\pi_{1}(|\mathcal{L}|)\right]$ module. If the action of $\pi_{1}(|\mathcal{L}|)$ on $M$ is p-solvable, then the inclusion of $B S$ in $|\mathcal{L}|$ induces a natural isomorphism

$$
H^{*}(|\mathcal{L}|, M) \stackrel{\cong}{\longrightarrow} H^{*}\left(\mathcal{F}^{c}, M\right) .
$$

Corollary 4.5 and Corollary 5.5 give good evidence for Conjecture 5.6 to be true.

The next section, which is a bit technical, is dedicated to give an example of Conjecture 5.6 where Corollary 5.5 doesn't apply (see Remark 6.7).

\section{The $p$-local structure of wreath products by $C_{p}$ : an example for Conjecture 5.6}

Let $G_{0}$ be a finite group, $S_{0}$ a Sylow $p$-subgroup of $G_{0}$ and $\left(S_{0}, \mathcal{F}_{0}, \mathcal{L}_{0}\right)$ be the associated $p$-local finite group. We are interested in the wreath product $G=G_{0} 2 C_{p}$, $S=S_{0} \prec C_{p}$ and the associated $p$-local finite group $(S, \mathcal{F}, \mathcal{L})$. By $[\mathbf{C L}$, Theorem 5.2 and Remark 5.3], we have that $|\mathcal{L}| \simeq\left|\mathcal{L}_{0}\right|\left\langle B C_{p}:=\left|\mathcal{L}_{0}\right|^{p} \times_{C_{p}} E C_{p}\right.$ and an extension $\left(\pi_{\mathcal{L}_{0}}\right)^{p} \rightarrow \pi_{\mathcal{L}} \rightarrow C_{p}$. In addition, we have a section $C_{p} \rightarrow \pi_{\mathcal{L}}$ coming from $* \imath B C_{p} \rightarrow$ $\left|\mathcal{L}_{0}\right| \succ B C_{p}$ and thus $\pi_{\mathcal{L}}=\pi_{\mathcal{L}_{0}}$ $C_{p}$.

We first give a lemma on strongly $p$-embedded subgroups. For a finite group $G$, a subgroup $H<G$ is strongly $p$-embedded, if $p|| H \mid$ and for each $x \in G \backslash H, H \cap x H x^{-1}$ has order prime to $p$.

Lemma 6.1. Let $G$ be a finite group, $G_{0} \leqslant G$ a subgroup of index a power of $p$. If $G$ contains a strongly p-embedded subgroup and $p|| G_{0} \mid$, then $G_{0}$ contains a strongly p-embedded subgroup.

Proof. Let $H$ be a strongly $p$-embedded subgroup of $G$. By [AKO, Proposition A.7], $H$ contains a Sylow $p$-subgroup of $G$ so, up to conjugacy, we can choose $H$ such that $H$ contains a Sylow $p$-subgroup of $G_{0}$. Hence $G_{0} \cap H$ contains a Sylow $p$-subgroup of $G_{0}$ and $p|| G_{0} \cap H \mid$. We will show that $G_{0} \cap H$ is a strongly $p$-embedded subgroup of $G_{0}$. 
As $[G: H]$ is prime to $p$ and $\left[G: G_{0}\right]$ is a power of $p, G_{0} \cap H$ is a proper subgroup of $G_{0}$.

It remains to show that, for each $x \in G_{0} \backslash G_{0} \cap H,\left(G_{0} \cap H\right) \cap x\left(G_{0} \cap H\right) x^{-1}$ has order prime to $p$. But $\left(G_{0} \cap H\right) \cap x\left(G_{0} \cap H\right) x^{-1} \leqslant H \cap x H x^{-1}$, thus, as $H$ is a strongly $p$-embedded subgroup of $G$, this last subgroup has order prime to $p$ for every $x \in G \backslash H$. In particular, for each $x \in G_{0} \backslash G_{0} \cap H,\left(G_{0} \cap H\right) \cap x\left(G_{0} \cap H\right) x^{-1}$ has order prime to $p$ and $G_{0} \cap H$ is a strongly $p$-embedded subgroup of $G_{0}$.

We give also a lemma on $\mathcal{F}_{1}$-essential subgroups for $\mathcal{F}_{1} \subseteq \mathcal{F}$ a subsystem of index a power of $p$. A proper subgroup $P<S$ is $\mathcal{F}$-essential if $P$ is $\mathcal{F}$-centric and fully normalized in $\mathcal{F}$, and if $\operatorname{Out}_{\mathcal{F}}(P)$ contains a strongly $p$-embedded subgroup.

Lemma 6.2. Let $(S, \mathcal{F}, \mathcal{L})$ be a $p$-local finite group and $\left(S_{1}, \mathcal{F}_{1}, \mathcal{L}_{1}\right)$ a p-local subgroup of index a power of $p$. If $P<S_{1}$ is $\mathcal{F}$-essential, then $P$ is $\mathcal{F}_{1}$-conjugate to an $\mathcal{F}_{1}$ essential subgroup and $P$ is $\mathcal{F}_{1}$-essential if and only if $P$ is fully normalized in $\mathcal{F}_{1}$.

Proof. Let $P<S_{1}$ be an $\mathcal{F}$-essential subgroup. Since $\mathcal{F}_{1}$ is saturated, $P$ is $\mathcal{F}_{1^{-}}$ conjugate to a subgroup of $S_{1}$ fully normalized in $\mathcal{F}_{1}$. If $P$ is $\mathcal{F}_{1}$-essential, it is, in particular, fully normalized in $\mathcal{F}_{1}$. Thus, it remains to prove that if $P$ is fully normalized in $\mathcal{F}_{1}$, then $P$ is $\mathcal{F}_{1}$-essential. For the remaining, we assume that $P$ is fully normalized in $\mathcal{F}_{1}$ and $\mathcal{F}$-essential.

$P$ is $\mathcal{F}_{1}$-centric: As $P$ is $\mathcal{F}$-centric, $C_{S}(Q)=Z(Q)$ for all $Q \in P^{\mathcal{F}}$. In particular, for all $Q \in P^{\mathcal{F}_{1}} \subseteq P^{\mathcal{F}}, C_{S_{1}}(Q)=Z(Q)$ and $P$ is $\mathcal{F}_{1}$-centric.

Out $_{\mathcal{F}_{1}}(P)$ contains a strongly $p$-embedded subgroup: Since $P$ is $\mathcal{F}$-essential, the group $\operatorname{Out}_{\mathcal{F}}(P)$ contains a strongly $p$-embedded subgroup. As $\mathcal{F}_{1}$ is a subsystem

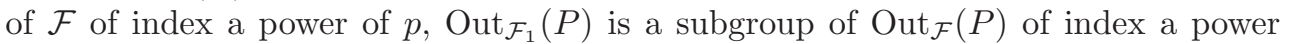
of $p$. Moreover, as $P$ is a proper subgroup of $S_{1}, P<N_{S_{1}}(P)$ and, as $P$ is $\mathcal{F}_{1}$ centric, every element of $N_{S_{1}}(P) \backslash Z(P)$ induces a non-trivial element in $\operatorname{Out}_{\mathcal{F}_{1}}(P)$.

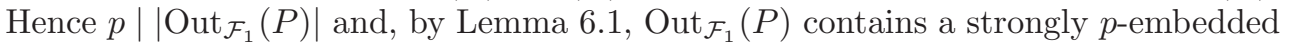
subgroup.

We can easily describe the essential subgroups of a product of fusion systems.

Lemma 6.3. Let $\left(S_{1}, \mathcal{F}_{1}, \mathcal{L}_{1}\right)$ and $\left(S_{2}, \mathcal{F}_{2}, \mathcal{L}_{2}\right)$ be $p$-local finite groups and set $S=$ $S_{1} \times S_{2}$ and $\mathcal{F}=\mathcal{F}_{1} \times \mathcal{F}_{2}$. The $\mathcal{F}$-essential subgroups of $S$ are of the form $Q_{1} \times S_{2}$ with $Q_{1}<S_{1} \mathcal{F}_{1}$-essential or $S_{1} \times Q_{2}$ with $Q_{2}>S_{2} \mathcal{F}_{2}$-essential.

Proof. Let $P \leqslant S$ be an $\mathcal{F}$-essential subgroup. By [AKO, Proposition I.3.3], $P$ is $\mathcal{F}$-centric and $\mathcal{F}$-radical. Thus, by [AOV, Lemma 3.1], $P=P_{1} \times P_{2}$ with $P_{i} \leqslant S_{i}$ and $P_{i} \mathcal{F}_{i}$-centric.

Remark also that, if we have two groups $G_{1}$ and $G_{2}$ such that $p$ divide $\left|G_{1}\right|$ and $\left|G_{2}\right|$ then $G_{1} \times G_{2}$ cannot contain a strongly $p$-embedded subgroup. To see that let $S_{i}$ be a Sylow $p$-subgroup of $G_{i}$ and set $H=\left\langle x \in G \mid x\left(S_{1} \times S_{2}\right) x^{-1} \cap S_{1} \times S_{2} \neq 1\right\rangle$. $H$ contains $G_{1} \times\{0\}$ and $\{0\} \times G_{2}$ so that $H=G$. Thus, by [AKO, Proposition A.7], this implies that $G$ has no strongly $p$-embedded subgroups.

We also have that $\operatorname{Out}_{\mathcal{F}}(P)=\operatorname{Out}_{\mathcal{F}_{1}}\left(P_{1}\right) \times \operatorname{Out}_{\mathcal{F}_{2}}\left(P_{2}\right)$. Hence, the only possibility for $P$ to be $\mathcal{F}$-essential is that $P_{1}=S_{1}$ and $P_{2}$ is $\mathcal{F}_{2}$-essential or the contrary.

Let $G_{0}$ be a finite group, $S_{0}$ a Sylow $p$-subgroup of $G_{0}$ and $\left(S_{0}, \mathcal{F}_{0}, \mathcal{L}_{0}\right)$ be the associated $p$-local finite group. We consider the wreath product $G=G_{0} \imath C_{p}, S=S_{0} \imath C_{p}$ 
and the associated $p$-local finite group $(S, \mathcal{F}, \mathcal{L})$. Here, for the direct computation, we will take the notation of Alperin and Fong $[\mathbf{A F}]$ : an element of $G$ will be represented by permutation matrix corresponding to the powers of $(1,2, \ldots, p)$ with entries in $G_{0}$ and the composition will follow the matrix product with the composition in $G_{0}$. Denote by $c \in G$ the element

$$
e \otimes P_{(1,2, \ldots, p)}=\left(\begin{array}{ccccc}
0 & 0 & \cdots & 0 & e \\
e & 0 & \cdots & 0 & 0 \\
\vdots & \ddots & & \vdots & \vdots \\
\vdots & & \ddots & 0 & 0 \\
0 & \cdots & \cdots & e & 0
\end{array}\right),
$$

where $e$ is the trivial element of $G_{0}$. Here, we are interested in the $\mathcal{F}$-essential subgroups.

Lemma 6.4. Let $P \leqslant S$ be an $\mathcal{F}$-essential subgroup.

$\left(E_{1}\right)$ If $P \leqslant S_{0}^{p}$, then either $P=S_{0}^{p}$ and $N_{G}(P)=N_{G_{0}}\left(S_{0}\right)$ 乙 $C_{p}$ or $P$ is $\mathcal{F}_{0}^{p}$-essential and $N_{G}(P)=N_{G_{0}^{p}}(P)$.

$\left(E_{2}\right)$ If $P \not \leq S_{0}^{p}$, then $P \cong_{\mathcal{F}} Q$ 乙 $C_{p}$ where $Q$ is $\mathcal{F}_{0}$-essential and we have $N_{G}(P) / P \cong$ $N_{G_{0}}(Q) / Q$ through the diagonal map $G_{0} \hookrightarrow G_{0}^{p}$.

Proof. Let $P \leqslant S$ be an $\mathcal{F}$-essential subgroup.

Assume first that $P \leqslant S_{0}^{p}$. If $P=S_{0}^{p}$ a direct calculation gives $N_{G}(P)=N_{G_{0}}\left(S_{0}\right)$ 2 $C_{p}$. Else, by Lemma 6.2 , we know that $P$ is $\mathcal{F}_{0}^{p}$-conjugate to an $\mathcal{F}_{0}^{p}$-essential subgroup $Q \leqslant S_{0}^{p}$. By Lemma 6.3 we have $N_{G}(Q) \leqslant G_{0}^{p}$ and, in particular, $N_{G}(Q)=N_{G_{0}^{p}}(Q)$. Thus, since $P$ is $\mathcal{F}_{0}^{p}$-conjugate to $Q$, we also have $N_{G}(P)=N_{G_{0}^{p}}(P)$ and, since $P$ is fully normalized in $\mathcal{F}$, it is fully normalized in $\mathcal{F}_{0}^{p}$. Hence, by Lemma $6.2, P$ is $\mathcal{F}_{0}$-essential.

Secondly, assume that $P \not \leq S_{0}^{p}$. As all choices of a splitting $C_{p} \rightarrow G$ are conjugate in $G$, we can assume that $P=\left\langle P_{0}, x\right\rangle$ where $P_{0}=P \cap S_{0}^{p}$ and $x=\left(\left(x_{1}, x_{2}, \ldots, x_{p}\right), c\right)$ is such that $x^{p} \in P_{0}$. Up to conjugation in $S_{0}<C_{p}$ we can assume that $x$ is of the form $((a, 1,1, \ldots, 1), c)$ where $a \in N_{S_{0}}(Q)$ where $Q$ is the projection of $P_{0}$ on the first factor. If we write $P_{0}^{(i)}$ the projection of $P_{0}$ on its $i$ th factor, as $x$ normalizes $P_{0}$, we have that $P_{0}^{(i)}=P_{0}^{(j)}$ for all $i, j$ and then $P_{0} \leqslant\left(P_{0}^{(1)}\right)^{p}=Q^{p}$.

Notice also that $N_{G}(P)=\left\langle N_{G_{0}^{p}}(P), x\right\rangle$. If $g=\left(g_{1}, \ldots, g_{p}\right) \in N_{G_{0}^{p}}(P)$, as $g$ normalizes $P \cap G_{0}^{p}=P_{0}$, we have, for all $i, g_{i} \in N_{G_{0}}(Q)$. Moreover, if we denote $h=$ $\left(h_{1}, \ldots, h_{p}\right)=g x g^{-1} x^{-1} \in P_{0}$, we have, for all $i, g_{i} h_{i}=g_{i-1}$ (with $\left.g_{0}=g_{p}\right)$. Therefore, there is $h^{\prime} \in Q^{p}$ such that $g=\left(g_{1}, g_{1}, \ldots, g_{1}\right) . h^{\prime} \in\left\langle N_{G_{0}}(Q) \otimes \operatorname{Id}, Q^{p}\right\rangle \leqslant N_{G}\left(Q^{p}\right)$. Hence, every automorphism $c_{g} \in \operatorname{Aut}_{\mathcal{F}}(P)$ can be extended to an automorphism of $\left\langle Q^{p}, x\right\rangle$. As $P$ is $\mathcal{F}$ essential, by [AKO, Proposition I.3.3], $P=\left\langle Q^{p}, x\right\rangle$. Now, $x^{p} \in Q^{p}$ implies that $a \in Q$ so $P=\left\langle Q^{p}, x\right\rangle=\left\langle Q^{p}, c\right\rangle=Q \imath C_{p}$.

Finally, direct computations give that

$$
C_{G}(P) \cong C_{G_{0}}(Q) \otimes \operatorname{Id}=\left\{\left(\begin{array}{cccc}
g & 0 & \cdots & 0 \\
0 & g & \ddots & \vdots \\
\vdots & \ddots & \ddots & 0 \\
0 & \cdots & 0 & g
\end{array}\right) ; g \in C_{G_{0}}(Q)\right\}
$$


and

$$
N_{G}(P) / P \cong N_{G_{0}}(Q) / Q \otimes \mathrm{Id} \cong N_{G_{0}}(Q) / Q
$$

In particular, as $P$ is $p$-centric, $Q$ is $G_{0}$-centric. Moreover, as $N_{G}(P) / P=\operatorname{Out}_{\mathcal{F}}(P)$ contains a strongly $p$-embedded subgroup, $\operatorname{Out}_{\mathcal{F}_{0}}(Q)=N_{G_{0}}(Q) / Q$ does as well. Up to conjugacy, we can also assume that $Q$ is fully normalized in $\mathcal{F}_{0}$ and thus $Q$ is $\mathcal{F}_{0}$-essential.

Let us now look at some cohomological results. Recall that for a group $G$, a subgroup $H \leqslant G$, and $M$ an $\mathbb{F}_{p}[H]$-module, we define the induced and coinduced $\mathbb{F}_{p}[G]$ module by,

$$
\operatorname{Ind}_{H}^{G}(M)=\mathbb{F}_{p}[G] \otimes_{\mathbb{F}_{p}[H]} M, \quad \operatorname{coInd}_{H}^{G}(M)=\operatorname{Hom}_{\mathbb{F}_{p}[H]}\left(\mathbb{F}_{p}[G], M\right) .
$$

Recall also that, when the index of $H$ in $G$ is finite, these two $\mathbb{F}_{p}[G]$-modules are isomorphic (by [We, Lemma 6.3.4]).

Lemma 6.5. Let $X$ be a $C W$ complex and denote by $G$ its fundamental group. If $X_{0}$ is a covering space of $X$ with fundamental group $G_{0} \unlhd G$ of finite index, then, for every $\mathbb{F}_{p}\left[G_{0}\right]$-module $M$, we have a natural isomorphism of $\mathbb{F}_{p}\left[G / G_{0}\right]$-modules,

$$
H^{*}\left(X_{0}, \operatorname{Ind}_{G_{0}}^{G}(M)\right) \cong H^{*}\left(X_{0}, M\right) \otimes_{\mathbb{F}_{p}} \mathbb{F}_{p}\left[G / G_{0}\right],
$$

where, on the right side, $G / G_{0}$ is only acting by translation on $\mathbb{F}_{p}\left[G / G_{0}\right]$.

Proof. This can be easily seen on the chain level. Let $\tilde{X}$ be the universal covering space of $X$. As $\mathbb{F}_{p}\left[G / G_{0}\right]$-modules, we have the following

$$
\operatorname{Hom}_{\mathbb{F}_{p}\left[G_{0}\right]}\left(C_{*}(\tilde{X}), \operatorname{Ind}_{G_{0}}^{G}(M)\right)=\bigoplus_{g \in\left[G / G_{0}\right]} \operatorname{Hom}_{\mathbb{F}_{p}\left[G_{0}\right]}\left(C_{*}(\tilde{X}), g \cdot M\right),
$$

where the action of $G / G_{0}$ is permuting the terms in the sum. But, each terms in the sum is isomorphic, as (trivial) $\mathbb{F}_{p}\left[G / G_{0}\right]$-modules, to $\operatorname{Hom}_{\mathbb{F}_{p}\left[G_{0}\right]}\left(C_{*}(\tilde{X}), M\right)$. Thus

$$
\operatorname{Hom}_{\mathbb{F}_{p}\left[G_{0}\right]}\left(C_{*}(\tilde{X}), \operatorname{Ind}_{G_{0}}^{G}(M)\right) \cong \operatorname{Hom}_{\mathbb{F}_{p}\left[G_{0}\right]}\left(C_{*}(\tilde{X}), M\right) \otimes_{\mathbb{F}_{p}} \mathbb{F}_{p}\left[G / G_{0}\right] .
$$

This induces the wanted isomorphism in cohomology.

Proposition 6.6. Let $G_{0}$ be a finite group and $\left(S_{0}, \mathcal{F}_{0}, \mathcal{L}_{0}\right)$ be the associated p-local finite group. Consider $G=G_{0} \prec C_{p}, S=S_{0} \succ C_{p}$ a Sylow p-subgroup of $G$ and $(S, \mathcal{F}, \mathcal{L})$ the associated p-local finite group. Let $M$ be an $\mathbb{F}_{p}\left[\pi_{\mathcal{L}_{0}}\right]$-module.

If $\delta_{S_{0}}$ induce natural isomorphisms

$$
H^{*}\left(\left|\mathcal{L}_{0}\right|, M\right) \cong H^{*}\left(\left(\mathcal{F}_{0}\right)^{c}, M\right),
$$

and

$$
H^{*}\left(\left|\mathcal{L}_{0}\right|^{p}, \operatorname{coInd} d_{\pi_{\mathcal{L}_{0}}^{p}}^{\pi_{\mathcal{L}}}\left(M^{\otimes p}\right)\right) \cong H^{*}\left(\left(\mathcal{F}_{0}^{p}\right)^{c}, \operatorname{coIn} d_{\pi_{\mathcal{L}_{0}}^{p}}^{\pi_{\mathcal{L}}}\left(M^{\otimes p}\right)\right)
$$

then $\delta_{S}$ induces a natural isomorphism

$$
H^{*}\left(|\mathcal{L}|, \operatorname{coIn} d_{\pi_{\mathcal{L}_{0}}^{p}}^{\pi_{\mathcal{L}}}\left(M^{\otimes p}\right)\right) \cong H^{*}\left(\mathcal{F}^{c}, \operatorname{coIn} d_{\pi_{\mathcal{L}_{0}}^{p}}^{\pi_{\mathcal{L}}^{p}}\left(M^{\otimes p}\right)\right)
$$


Proof. Write $N=\operatorname{coInd}_{\pi_{\mathcal{L}_{0}}^{p}}^{\pi_{\mathcal{L}}}\left(M^{\otimes p}\right)$ and, for $i \in\{1,2\}$, denote by $H^{*}\left(\mathcal{F}^{E_{i}}, N\right)$ the stable elements of $H^{*}(S, N)$ under the full subcategory of $\mathcal{F}$ with objects $S$ and all the subgroups of $S$ of type $\left(E_{i}\right)$ defined in Lemma 6.4 .

By the Mackey Formula,

$$
\operatorname{Res}_{Q \mathcal{L} C_{p}}^{\pi_{\mathcal{L}}} \operatorname{Ind}_{\pi_{\mathcal{L}_{0}}^{p}}^{\pi_{\mathcal{L}}}=\operatorname{Ind}_{Q^{p}}^{Q \imath C_{p}} \operatorname{Res}_{Q^{p}} \pi_{\mathcal{L}_{0}}^{p}
$$

Thus by Shapiro's Lemma (see for example [Ev, Proposition 4.1.3]) and the Kunneth Formula, for every $P=Q \imath C_{p}$ of type $\left(E_{2}\right)$, we have a natural isomorphism $H^{*}(Q \imath$ $\left.C_{p}, N\right) \cong H^{*}\left(Q^{p}, M^{\otimes p}\right) \cong H^{*}(Q, M)^{\otimes p}$ and, by the computation of normalizers in Lemma 6.4,

$$
H^{*}\left(Q \succ C_{p}, N\right)^{\operatorname{Aut}_{\mathcal{F}}\left(Q \succ C_{p}\right)} \cong\left(H^{*}(Q, M)^{\operatorname{Aut}_{\mathcal{F}_{0}}(Q)}\right)^{\otimes p} .
$$

Hence, applying this to all the subgroups of type $\left(E_{2}\right)$ and, by naturality of the Shapiro isomorphisms, we have that,

$$
H^{*}\left(\mathcal{F}^{E_{2}}, N\right) \cong H^{*}\left(\mathcal{F}_{0}^{c}, M\right)^{\otimes p} .
$$

On the other hand, by [CL, Theorem 5.2 and Remark 5.3], $\left|\mathcal{L}_{0}\right|^{p}$ has the homotopy type of a covering space of $|\mathcal{L}|$ with covering group $C_{p}$. Then, if we denote by $X$ the universal covering space of $|\mathcal{L}|$ (which is also the universal covering space of $\left|\mathcal{L}_{0}\right|^{p}$ ), we have the following isomorphism on the chain level (because Res and coInd are adjoint functors)

$$
\operatorname{Hom}_{\mathbb{Z}_{(p)}\left[\pi_{\mathcal{L}_{0}}^{p}\right]}\left(C_{*}(X), M^{\otimes p}\right) \cong \operatorname{Hom}_{\mathbb{Z}_{(p)}\left[\pi_{\mathcal{L}}\right]}\left(C_{*}(X), N\right),
$$

which is analogue to the Shapiro isomorphism (see [Ev, Proposition 4.1.3]). By the Kunneth Formula, it gives us the following isomorphism on cohomology

$$
H^{*}\left(\left|\mathcal{L}_{0}\right|, M\right)^{\otimes p} \cong H^{*}(|\mathcal{L}|, N)
$$

and give the following commutative diagram:

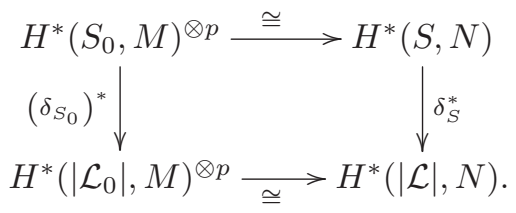

Thus $\delta_{S}$ induces an isomorphism

$$
H^{*}\left(\mathcal{F}^{E_{2}}, N\right) \cong H^{*}\left(\mathcal{F}_{0}^{c}, M\right)^{\otimes p} \cong H^{*}\left(\left|\mathcal{L}_{0}\right|, M\right)^{\otimes p} \cong H^{*}(|\mathcal{L}|, N) .
$$

Secondly, by factoring the Shapiro isomorphism (see [Ev, Proposition 4.1.3]), the inclusion of $S_{0}^{p}$ in $S$ induces an injection $H^{*}(S, N) \hookrightarrow H^{*}\left(S_{0}^{p}, N\right)$. Hence

$$
H^{*}\left(\mathcal{F}^{E_{1}}, N\right) \cong H^{*}\left(\left(\mathcal{F}_{0}^{p}\right)^{c}, N\right)^{C_{p}} \leqslant H^{*}\left(S_{0}^{p}, N\right) .
$$

By assumption, $\delta_{S_{0}^{p}}$ induces an isomorphism

$$
H^{*}\left(\left(\mathcal{F}_{0}^{p}\right)^{c}, N\right) \cong H^{*}\left(\left|\mathcal{L}_{0}\right|^{p}, N\right) .
$$

Moreover, by Lemma 6.5, this last term is isomorphic to $H^{*}\left(\left|\mathcal{L}_{0}\right|^{p}, M^{\otimes p}\right) \otimes \mathbb{F}_{p}\left[C_{p}\right]$ and, in particular, it is a projective $\mathbb{F}_{p}\left[C_{p}\right]$-module. 
Consider now the Serre spectral sequence associated to the fibration sequence

$$
\left|\mathcal{L}_{0}\right|^{p} \longrightarrow|\mathcal{L}| \longrightarrow B C_{p},
$$

with coefficients in $N$. The $E_{2}$ page is the following,

$$
E_{2}^{s, t}=H^{s}\left(C_{p}, H^{t}\left(\left|\mathcal{L}_{0}\right|^{p}, N\right)\right)
$$

and, by projectivity of $H^{t}\left(\left|\mathcal{L}_{0}\right|^{p}, N\right)$, the $E_{2}$ page is concentrated in the 0th column. Hence, we have that, $H^{*}\left(\left|\mathcal{L}_{0}\right|^{p}, N\right)^{C_{p}}=E_{2}^{0, *} \cong H^{*}(|\mathcal{L}|, N)$.

In conclusion,

$$
H^{*}\left(\mathcal{F}^{c}, N\right)=H^{*}\left(\mathcal{F}^{E_{1}}, N\right) \cap H^{*}\left(\mathcal{F}^{E_{2}}, N\right) \cong H^{*}(|\mathcal{L}|, N)
$$

and the theorem follows.

This proposition is a bit technical but we will use it in a specific case. Consider $p=5$, the group $G_{0}=G L_{20}\left(F_{2}\right)$, the wreath product $G=G_{0} \succ C_{5}$ and $\left(S_{0}, \mathcal{F}_{0}, \mathcal{L}_{0}\right)$ and $(S, \mathcal{F}, \mathcal{L})$ the associated 5 -local finite groups. By [Ru, Theorem 6.3], we know that $\left(S_{0}, \mathcal{F}_{0}, \mathcal{L}_{0}\right)$ admits a 5 -local subgroup of index 4 which is exotic $\left(S_{e}, \mathcal{F}_{e}, \mathcal{L}_{e}\right)$ and that we have a fibration sequence

$$
\left|\mathcal{L}_{e}\right| \longrightarrow\left|\mathcal{L}_{0}\right| \longrightarrow B C_{4} .
$$

In particular, we have $\pi_{\mathcal{L}} / \pi_{\mathcal{L}_{e}}^{5}=C_{4} \curlywedge C_{5}$ and we can be interested in comparing $H^{*}(|\mathcal{L}|, N)$ and $H^{*}\left(\mathcal{F}^{c}, N\right)$ for

$$
N=\mathbb{F}_{5}\left[C_{4} \curlywedge C_{5}\right]=\operatorname{Ind}_{\pi_{\mathcal{L}_{0}}^{5}}^{\pi_{\mathcal{L}}}\left(M^{\otimes 5}\right) \cong \operatorname{coInd}_{\pi_{\mathcal{L}_{0}}^{5}}^{\pi_{\mathcal{L}}}\left(M^{\otimes 5}\right)
$$

(the action factors through a finite group) with $M=\mathbb{F}_{5}\left[C_{4}\right]$.

By Corollary 4.4, we have that $\delta_{S_{0}}$ and $\delta_{S_{0}^{p}}$ induce natural isomorphisms

$$
\begin{gathered}
H^{*}\left(\left|\mathcal{L}_{0}\right|, M\right) \cong H^{*}\left(\left(\mathcal{F}_{0}\right)^{c}, M\right) \text { and } \\
H^{*}\left(\left|\mathcal{L}_{0}\right|^{5}, \operatorname{coInd}_{\pi_{\mathcal{L}_{0}}^{5}}^{\pi_{\mathcal{L}}}\left(M^{\otimes 5}\right)\right) \cong H^{*}\left(\left(\mathcal{F}_{0}^{5}\right)^{c}, \operatorname{coInd}_{\pi_{\mathcal{L}_{0}}^{5}}^{\pi_{\mathcal{L}}}\left(M^{\otimes 5}\right)\right),
\end{gathered}
$$

(for the second isomorphism, notice that $\left|\mathcal{L}_{0}\right|^{5}$ has the homotopy type of a linking system associated to $\mathcal{F}_{0}^{5}$ by $[\mathbf{C L}$, Proposition 2.17]). Hence, all the hypothesis of Proposition 6.6 are satisfied and

$$
H^{*}(|\mathcal{L}|, N) \cong H^{*}\left(\mathcal{F}^{c}, N\right)
$$

Remark 6.7. This gives us an example of isomorphism between the cohomology of $|\mathcal{L}|$ and the stable elements when the action factors through a $p$-solvable group which cannot be recovered by a previous result. Notice that, even if the fusion system $\mathcal{F}$ is realizable, as $\mathcal{F}_{e}$ is exotic, we cannot find a group $G$ with $S \in \operatorname{Syl}_{p}(G)$ such that $G$ acts on $M$ in the same way as asked in Section 5. This example gives us some additional evidence for Conjecture 5.6.

\section{References}

[5a1] C. Broto, N. Castellana, J. Grodal, R. Levi and B. Oliver, Subgroup families controlling p-local finite groups, Proc. Lond. Math. Soc. (3) 91 (2005), no. $2,325-354$. 
[5a2] C. Broto, N. Castellana, J. Grodal, R. Levi and B. Oliver, Extensions of $p$ local finite groups, Trans. Amer. Math. Soc. 359 (2007), no. 7, 3791-3858.

[AF] J.L. Alperin and P. Fong, Weights for symmetric and general linear groups, J. Algebra 131 (1990), no. 1, 2-22.

[AKO] M. Aschbacher, R. Kessar and B. Oliver, Fusion Systems in Algebra and Topology, volume 391 of London Mathematical Society Lecture Note Series, Cambridge University Press, Cambridge, 2011.

[AOV] K.K.S. Andersen, B. Oliver and J. Ventura, Reduced, tame and exotic fusion systems, Proc. Lond. Math. Soc. (3) 105 (2012), no. 1, 87-152.

[BLO1] C. Broto, R. Levi and B. Oliver, Homotopy equivalences of $p$-completed classifying spaces of finite groups, Invent. Math. 151 (2003), no. 3, 611-664.

[BLO2] C. Broto, R. Levi and B. Oliver, The homotopy theory of fusion systems, J. Amer. Math. Soc. 16 (2003), no. 4, 779-856.

[CE] H. Cartan and S. Eilenberg, Homological Algebra, Princeton Landmarks in Mathematics, Princeton University Press, Princeton, NJ, 1999.

[CL] N. Castellana and A. Libman, Wreath products and representations of p-local finite groups, Adv. Math. 221 (2009), no. 4, 1302-1344.

[Ch] A. Chermak, Fusion systems and localities, Acta Math. 211 (2013), no. 1, 47-139.

[Ev] L. Evens, The Cohomology of Groups, Oxford Mathematical Monographs, The Clarendon Press, Oxford University Press, New York, 1991.

[Gr] J. Grodal, Higher limits via subgroup complexes, Ann. of Math. (2) 155 (2002), no. 2, 405-457.

[LR] R. Levi and K. Ragnarsson, p-local finite group cohomology, Homology Homotopy Appl. 13 (2011), no. 1, 223-257.

[Mo1] R. Molinier, Cohomology with twisted coefficients of the classifying space of a fusion system, Topology Appl. 212 (2016), 1-18.

[O4] B. Oliver, Extensions of linking systems and fusion systems, Trans. Amer. Math. Soc. 362 (2010), no. 10, 5483-5500.

[OV1] B. Oliver and J. Ventura, Extensions of linking systems with $p$-group kernel, Math. Ann. 338 (2007), no. 4, 983-1043.

[Ru] A. Ruiz, Exotic normal fusion subsystems of general linear groups, J. Lond. Math. Soc. (2) 76 (2007), no. 1, 181-196.

[We] C.A. Weibel, An Introduction to Homological Algebra, volume 38 of Cambridge Studies in Advanced Mathematics, Cambridge University Press, Cambridge, 1994.

Rémi Molinier molinier@ksu.edu

Department of Mathematics, Kansas State University, 138 Cardwell Hall, Manhattan, KS 66506, USA 